\title{
FINITE BASIS THEOREMS FOR RELATIVELY CONGRUENCE-DISTRIBUTIVE QUASIVARIETIES
}

\author{
DON PIGOZZI
}

\begin{abstract}
Q}$ is any quasivariety. A congruence relation $\Theta$ on a member $\mathbf{A}$ of $\mathcal{Q}$ is a $\mathcal{Q}$-congruence if $\mathbf{A} / \Theta \in \mathcal{Q}$. The set $\operatorname{Con}_{\mathcal{Q}} \mathbf{A}$ of all $\mathcal{Q}$ congruences is closed under arbitrary intersection and hence forms a complete lattice $\operatorname{Con}_{\mathcal{Q}} \mathbf{A}$. $\mathcal{Q}$ is relatively congruence-distributive if $\operatorname{Con}_{\mathcal{Q}} \mathbf{A}$ is distributive for every $\mathbf{A} \in \mathcal{Q}$. Relatively congruence-distributive quasivarieties occur naturally in the theory of abstract data types. $\mathcal{Q}$ is finitely generated if it is generated by a finite set of finite algebras. The following generalization of Baker's finite basis theorem is proved. Theorem I. Every finitely generated and relatively congruence-distributive quasivariety is finitely based. A subquasivariety $\mathcal{R}$ of an arbitrary quasivariety $\mathcal{Q}$ is called a relative subvariety of $\mathcal{Q}$ if it is of the form $\mathcal{V} \cap \mathcal{Q}$ for some variety $\mathcal{V}$, i.e., a base for $R$ can be obtained by adjoining only identities to a base for $\mathcal{Q}$. Theorem II. Every finitely generated relative subvariety of a relatively congruence-distributive quasivariety is finitely based. The quasivariety of generalized equality-test algebras is defined and the structure of its members studied. This gives rise to a finite algebra whose quasi-identities are finitely based while its identities are not. Connections with logic and the algebraic theory of data types are discussed.
\end{abstract}

Introduction. Let $\mathcal{Q}$ be any quasivariety, and let $\mathbf{A}$ be any algebra of the same language type as $\mathcal{Q}$ (but not necessarily itself a member of $\mathcal{Q}$ ). A congruence relation $\Theta$ on $\mathbf{A}$ is called a $\mathcal{Q}$-congruence if $\mathbf{A} / \Theta \in \mathcal{Q}$. The set $\operatorname{Con}_{\mathcal{Q}} \mathbf{A}$ of all $\mathcal{Q}$-congruences is closed under arbitrary intersection and hence forms a complete lattice $\operatorname{Con}_{\mathcal{Q}} \mathbf{A}$. $\mathcal{Q}$ is relatively congruence-distributive if $\operatorname{Con}_{\mathcal{Q}} \mathbf{A}$ is distributive for every $\mathbf{A} \in \mathcal{Q}$. A subquasivariety $R$ of a quasivariety $\mathcal{Q}$ is called a relative subvariety of $\mathcal{Q}$ if it is of the form $\mathcal{V} \cap \mathcal{Q}$ for some variety $\mathcal{V}$, i.e., a base for $R$ can be obtained by adjoining only identities to a base for $\mathcal{Q}$.

The main purpose of this paper is to prove the following two quasivariety analogues of Baker's finite basis theorem [2]. A quasivariety $\mathcal{Q}$ is finitely generated if it is generated by a finite set of finite algebras. Any set of quasi-identities that defines $\mathcal{Q}$ is called a base for $\mathcal{Q}$. All algebras are assumed to have only finitely many fundamental operations.

THEOREM I. Every finitely generated and relatively congruence-distributive quasivariety is finitely based.

THEOREM II. Let $\mathcal{Q}$ be a relatively congruence-distributive quasivariety. Then every finitely generated relative subvariety of $\mathcal{Q}$ is finitely based.

Received by the editors June 16, 1988.

1980 Mathematics Subject Classification (1985 Revision). Primary 08C15; Secondary 03C05, 08B05, $68 \mathrm{Q} 65$.

Key words and phrases. Quasivariety, finite basis theorem, Baker's theorem, relatively congruencedistributive, abstract data type. 
If $\mathcal{Q}$ is a variety and $\mathbf{A} \in \mathcal{Q}$, then every congruence on $\mathbf{A}$ is a $\mathcal{Q}$-congruence, and hence $\operatorname{Con}_{\mathcal{Q}} \mathbf{A}=\mathbf{C o n} \mathbf{A}$. So for varieties, relative congruence-distributivity coincides with congruence-distributivity in the usual sense. Baker's theorem is thus an immediate corollary of Theorem I since, by Jónsson's lemma [19], any finitely generated congruence-distributive variety is also finitely generated as a quasivariety.

The property of being relatively congruence-distributive is not inherited by subquasivarieties. In particular, a quasivariety may generate a (relatively) congruencedistributive variety without itself having the property, and vice-versa; examples of both kinds are easy to find. The relatively congruence-distributive quasivarieties that generate a congruence-distributive variety have been characterized by Czelakowski and Dziobiak [12]. This characterization, and other general methods for establishing relative congruence-distributivity, are discussed at the beginning of $\S 7$.

Relative congruence-distributivity occurs naturally among the heterogeneous (many-sorted) algebras that arise in the theory of abstract data types. The homogeneous analogue of one such class of algebras is investigated in some detail in §7. An algebra is called an equality-test algebra if it contains the two-element Boolean algebra as a subreduct, and an equality-test operation $e q$ such that $e q(a, b)$ equals 1 if $a=b$ and 0 otherwise. A member of the quasivariety generated by all equality-test algebras of a given type is called a generalized equality-test algebra. The quasivariety itself is denoted by $\mathcal{G E} T$. It is congruence-distributive in the relative but not the absolute sense. In $\S 7$ we give a finite axiomatization for $\mathcal{G} \mathcal{E} T$ and study the structure of its members. One result is that the quasivariety generated by any equality-test algebra is a relative subvariety of $\mathcal{G E} \tau$ and hence relatively congruence-distributive.

Theorems I and II are closely related, in fact each is an easy corollary of the other. But they can apply in quite different situations. Let $\mathcal{K}$ be any class of algebras and let $Q v \mathcal{K}$ be the quasivariety generated by $K$. When $K$ is included in a quasivariety $\mathcal{Q}$, let $V a_{\mathcal{Q}} \mathcal{K}$ be the relative subvariety of $\mathcal{Q}$ generated by $\mathcal{K}$. In general $Q v \mathcal{K}$ is strictly smaller that $V a_{\mathcal{Q}} K$, and one may be relatively congruence-distributive while the other is not. The difference between the two theorems can be clearly seen by comparing their syntactical forms. To simplify matters let us consider a single finite algebra $\mathbf{A}$. If $\mathbf{A}$ is contained in a relatively congruence-distributive quasivariety $\mathcal{Q}$, then by Theorem II the identities of $\mathbf{A}$ are logical consequences of a finite set of quasi-identities of $\mathbf{A}$. However, if $Q v \mathbf{A}$ itself is relatively congruencedistributive, then the quasi-identities of $\mathbf{A}$ themselves are finitely based. There are situations where Theorem II applies and Theorem I does not. Belkin [4] has found a finite lattice $\mathbf{L}$ whose quasi-identities are not finitely based. But $\mathbf{L}$ is contained in a relatively congruence-distributive quasivariety, the variety of all lattices for example, so by Theorem II the identities of $\mathbf{L}$ are consequences of a finite set of its quasi-identities. Of course Baker's theorem itself applies in this case and gives a much stronger result than II.

In $\S 7$ we construct a finite equality-test algebra $\mathbf{E}$ such that $V a \mathbf{E}$ is not congruence-distributive; in fact $V a \mathbf{E}$ is not finitely based. Thus, since $Q v \mathbf{E}$ is finitely based by Theorem I, $\mathbf{E}$ has just the opposite property of Belkin's lattice: its quasiidentities are finitely based while its identities are not. 
Outline of paper. A somewhat weaker version of Theorem II is proved in $\S 3$ (Theorem 3.1). It is the exact algebraic analogue of a metalogical result of [9], and its proof is almost a direct translation; see also Dziobiak [15, Theorem 4.1]. A weaker version of Theorem I is then proved in $\S 6$ (Theorem 6.1). Its proof combines the methods of the proof of Theorem 3.1 with those of a result of Czelakowski and Dziobiak [12, Theorem 3.4]. The two weaker theorems are combined to give Theorem I in its full force in $\S 6$, and Theorem II is then obtained as a corollary.

In $\S \S 1$ and 2 we outline the basic facts we shall need about quasivarieties, relative subvarieties, and relative congruence-distributivity. $\S \S 4$ and 5 are devoted to establishing certain technical lemmas used in the proof of Theorem 6.1. The various methods available for establishing relative congruence-distributivity are described in $\S 7$, and equality-test and generalized equality-test algebras are investigated. Connections with the algebraic theory of data structures, including the specification problem, are discussed in $\S 8$.

In $\S 9$ we discuss the backgrounds of Theorems I and II and their connection with logic. We also give a number of open problems.

The author would like to express his gratitude to the members and staff of the Section of Logic of the Polish Academy of Sciences, especially Professors Ryzard Wójcicki, Janus Czelakowski, and Wiesław Dziobiak. Their kind hospitality during my visit to Lódź during the Summer of 1986, and the generosity with which they shared their latest results, contributed immeasurably to the production of this paper. Thanks are also due the faculty and staff of the Department of Mathematics of the University of Illinois at Chicago for their support during the 1986-87 academic year.

1. Quasivarieties and relative subvarieties. As mentioned in the Introduction, all language types considered in this paper are finite.

By a quasi-equation we mean a formula of the form

$$
\kappa_{0}(\bar{x}) \approx \lambda_{0}(\bar{x}) \wedge \cdots \wedge \kappa_{k-1}(\bar{x}) \approx \lambda_{k-1}(\bar{x}) \rightarrow \pi(\bar{x}) \approx \rho(\bar{x}),
$$

where $\kappa_{0}, \ldots, \kappa_{k-1}, \lambda_{0}, \ldots, \lambda_{k-1}, \pi, \rho$ are arbitrary terms, and the sequence $\bar{x}:=$ $x_{0}, \ldots, x_{m-1}$ includes every variable occurring in at least one of the terms. $k=0$ is possible so every equation qualifies as a quasi-equation. " $\approx$ " denotes the equality symbol in our formal languages. We use "=" for identity and ":=" to indicate a definition. A universally quantified quasi-equation is a quasi-identity. Following common practice we usually do not explicitly write the universal quantifiers on quasi-identities, leaving it to context to distinguish them from quasi-equations.

Any class of algebras defined by a set of quasi-identities is called a quasivariety. If $\mathcal{Q}$ is a quasivariety, any set $\Gamma$ of quasi-identities defining $\mathcal{Q}$ is called a base for $\mathcal{Q}$, and we write $\mathcal{Q}:=\operatorname{Mod} \Gamma$. Any class $\mathcal{K}$ of algebras such that $\mathcal{Q}$ is the smallest quasivariety including $\mathcal{K}$ is said to generate $\mathcal{Q}$, and we write $\mathcal{Q}:=Q v \mathcal{K}$. ( VaK denotes the variety generated by $K$.) By a well-known result of $\mathrm{A}$. $\mathrm{Mal}^{\prime} \mathrm{cev}$ (see for instance Burris and Sankappanavar [10]), $Q v K=\operatorname{ISPP}_{U} \mathcal{K}$ for any class $\mathcal{K}$ of algebras, where $\mathrm{I}, \mathrm{S}, \mathrm{P}$, and $\mathrm{P}_{\mathrm{U}}$ respectively denote the operations of forming isomorphic images, subalgebras, direct products, and ultraproducts. $\mathcal{Q}$ is finitely generated if $\mathcal{Q}=Q v \mathcal{K}$ for some finite set $\mathcal{K}$ of finite algebras.

Let $R$ be a binary relation on an algebra $\mathbf{A}$. $R$ is closed under the quasiequation (1) if, for any $\bar{a} \in A^{n}, R$ contains $\left\langle\pi^{\mathbf{A}}(\bar{a}), \rho^{\mathbf{A}}(\bar{a})\right\rangle$ whenever it contains 
$\left\langle\kappa_{i}^{\mathbf{A}}(\bar{a}), \lambda_{i}^{\mathbf{A}}(\bar{a})\right\rangle$ for every $i<n . R$ is a congruence relation on $\mathbf{A}$ iff it is closed under the quasi-equations $x \approx x, x \approx y \rightarrow y \approx x, x \approx y \wedge y \approx z \rightarrow x \approx z$, and, for each operation symbol $\omega$,

$$
x_{0} \approx y_{0} \wedge \cdots \wedge x_{m-1} \approx y_{m-1} \rightarrow \omega x_{0} \cdots x_{m-1} \approx \omega y_{0} \cdots y_{m-1} .
$$

If $\Phi$ is a congruence, then $\Phi$ is closed under a quasi-equation $\psi(\bar{x})$ iff $\mathbf{A} / \Phi \vDash \psi(\bar{x})$ (or, more explicitly, $\mathbf{A} / \Phi \vDash \forall \bar{x} \psi(\bar{x})$ ).

Let $\mathcal{Q}$ be a quasivariety and $\mathbf{A}$ any algebra, not necessarily in $\mathcal{Q}$. A congruence $\Phi$ on $\mathbf{A}$ is called a $\mathcal{Q}$-congruence if $\mathbf{A} / \Phi \in \mathcal{Q}$. The set of all $\mathcal{Q}$-congruences of $\mathbf{A}$ is denoted by $\operatorname{Con}_{\mathcal{Q}} \mathbf{A}$. $\operatorname{Con}_{\mathcal{Q}} \mathbf{A}$ always contains the universal congruence $\nabla_{A}$, and it contains the identity congruence $\Delta_{A}$ iff $\mathbf{A} \in \mathcal{Q}$. Let $\Gamma$ be any base for $\mathcal{Q}$. Clearly $\Phi$ is a $\mathcal{Q}$-congruence iff it is closed under each quasi-equation in $\Gamma$. From this observation it follows at once that $\operatorname{Con}_{\mathcal{Q}} \mathbf{A}$ is closed under arbitrary intersection and the union of directed sets. (This can also be seen from the fact that $\mathcal{Q}$ is closed under subdirect and reduced products.) Thus $\operatorname{Con}_{\mathcal{Q}} \mathbf{A}$ forms the universe of an algebraic lattice $\mathbf{C o n}_{\mathcal{Q}} \mathbf{A}$.

A quasivariety $\mathcal{Q}$ is relatively congruence-distributive if $\mathrm{Con}_{\mathcal{Q}} \mathbf{A}$ is distributive for each $\mathbf{A} \in \mathcal{Q}$.

We define

$$
\Theta_{\mathcal{Q}}(X):=\bigcap\left\{\Phi \in \operatorname{Con}_{\mathcal{Q}} \mathbf{A}: X \subseteq \Phi\right\}
$$

for every $X \subseteq A \times A$. The finitely generated $\mathcal{Q}$-congruences are exactly the compact elements of $\operatorname{Con}_{\mathcal{Q}} \mathbf{A}$. For any $a, b \in A, \Theta_{\mathcal{Q}}(a, b):=\Theta_{\mathcal{Q}}(\{\langle a, b\rangle\})$ is the principal $\mathcal{Q}$-congruence generated by the pair $a, b$.

A nontrivial algebra $\mathbf{A} \in \mathcal{Q}$ is said to be (finitely) subdirectly irreducible relative to $\boldsymbol{Q}$ if it is not isomorphic to a subdirect product of a (finite) system $\mathbf{B}_{i}, i \in I$, of algebras in $\mathcal{Q}$, unless at least one of the $\mathbf{B}_{i}$ is isomorphic to $\mathbf{A}$; in symbols, $\mathbf{A} \cong \mathbf{A}^{\prime} \subseteq \mathrm{SD} \prod_{i \in I} \mathbf{B}_{i}$ with $\mathbf{B}_{i} \in \mathcal{Q}$ (and $I$ finite) only if $\mathbf{A} \cong \mathbf{B}_{i}$ for some $i$. When $\mathcal{Q}$ is clear from context we say that $\mathbf{A}$ is relatively (finitely) subdirectly irreducible.

It is easily seen that $\mathbf{A} \in \mathcal{Q}$ is relatively (finitely) subdirectly irreducible iff $\Delta_{\mathbf{A}}$ is (finitely) meet irreducible in $\operatorname{Con}_{\mathcal{Q}} \mathbf{A}$. Since $\operatorname{Con}_{\mathcal{Q}} \mathbf{A}$ is algebraic, every $\mathcal{Q}$-congruence on $\mathbf{A}$ is the meet of finitely meet irreducible $\mathcal{Q}$-congruences. Thus we get the following relativized version of Birkhoff's subdirect representation theorem [5] for varieties.

THEOREM 1.1. Let $\mathcal{Q}$ be a quasivariety. Then every algebra in $\mathcal{Q}$ is isomorphic to a subdirect product of relatively subdirectly irreducible members of $\mathcal{Q}$.

The class of all relatively (finitely) subdirectly irreducible algebras of $\mathcal{Q}$ is denoted by $\mathcal{Q}_{\mathrm{RSI}}\left(\mathcal{Q}_{\mathrm{RFSI}}\right)$. Every absolutely (finitely) subdirectly irreducible member of $\mathcal{Q}$ is relatively (finitely) subdirectly irreducible. More generally, if $\mathcal{Q}$ is a subquasivariety of a quasivariety $\mathcal{Q}^{\prime}$, then $\mathcal{Q} \cap \mathcal{Q}_{\mathrm{RSI}}^{\prime} \subseteq \mathcal{Q}_{\mathrm{RSI}}$ and $\mathcal{Q} \cap \mathcal{Q}_{\mathrm{RFSI}}^{\prime} \subseteq \mathcal{Q}_{\mathrm{RFSI}}$. In general these inclusions are not equalities unless $\mathcal{Q}$ is a relative subvariety of $\mathcal{Q}^{\prime}$; see Lemma 1.4(2) below.

A quasivariety analogue of Jónsson's lemma [19] also holds. Actually two versions hold, one for quasivarieties and one for relative subvarieties. Both versions are easy consequences of the following lemma. Recall that an element $a$ of a lattice is meet prime if $b \wedge c \leq a$ implies $b \leq a$ or $c \leq a$ for all $b, c$. 
LEMMA 1.2. Let $\mathcal{Q}$ be a quasivariety and $\mathbf{C}_{i}$ with $i \in I$ any nonempty system of algebras in $\mathcal{Q}$. Let $\mathbf{B}$ be a nontrivial subalgebra of $\prod_{i \in I} \mathbf{C}_{i}$, and let $\Phi$ be a meet prime element of $\mathbf{C o n}_{\mathcal{Q}} \mathbf{B}$. Then there exists an ultrafilter $U$ on $I$ such that $\Psi_{J} \cap B^{2} \subseteq \Phi$ for every $J \in U$, where

$$
\Psi_{J}:=\left\{\langle a, b\rangle \in \prod_{i \in I} C_{i}: a_{j}=b_{j} \text { for all } j \in J\right\} .
$$

PrOOF. If $\Phi$ is the universal congruence, the conclusion if obvious. So assume $\Phi \neq \nabla_{B}$. Let $\mathbf{C}:=\prod_{i \in I} \mathbf{C}_{i}$. Observe that, for each $J \subseteq I, \Psi_{J}$ is the relationkernel of the projection of $\mathbf{C}$ onto $\prod_{j \in J} \mathbf{C}_{j}$. Thus $\mathbf{C} / \Psi_{J} \cong \prod_{j \in J} \mathbf{C}_{j} \in \mathcal{Q}$, and hence $\Psi_{J} \in$ Con $_{\mathcal{Q}} \mathrm{C}$ for every $J \subseteq I$.

Let $\bar{J}$ be the complement of $J$ in $I$. Define

$$
F:=\left\{J \subseteq I: \Psi_{\bar{J}} \cap B^{2} \nsubseteq \Phi\right\} .
$$

Then $I \in F$ since $\Psi_{\bar{I}}=\nabla_{C}$, and $\nabla_{C} \cap B^{2}=\nabla_{B} \nsubseteq \Phi$ by assumption. Also $J \subseteq J^{\prime}$ implies $\Psi_{\bar{J}} \subseteq \Psi_{\overline{J^{\prime}}}$, so $J^{\prime} \in F$ whenever $J \in F$ and $J \subseteq J^{\prime}$. Suppose $J, J^{\prime} \in F$. Then $\Psi_{\bar{J}} \cap B^{2}, \Psi_{\overline{J^{\prime}}} \cap B^{2} \notin \Phi$. Thus $\Psi_{\bar{J}} \cap \Psi_{\overline{J^{\prime}}} \cap B^{2} \nsubseteq \Phi$ since $\Phi$ is meet prime by hypothesis. But $\Psi_{\overline{J \cap J^{\prime}}}=\Psi_{\bar{J} \cup \overline{J^{\prime}}}=\Psi_{\bar{J}} \cap \Psi_{\overline{J^{\prime}}}$. Hence $J \cap J^{\prime} \in F$, and $F$ is a filter. $F$ is proper since $\Psi_{\bar{\varnothing}} \cap B^{2}=\Psi_{I} \cap B^{2}=\Delta_{C} \cap B^{2}=\Delta_{B} \subseteq \Phi$.

Let $U$ be any ultrafilter extending $F$, and let $J \in U$. Then $\bar{J} \notin U$ and hence $\bar{J} \notin F$, and so, by definition of $F, \Psi_{J} \cap B^{2} \subseteq \Phi$.

The next theorem is the relativized Jónsson lemma for quasivarieties.

THEOREM $1.3[12]$. Let $\mathcal{K}$ be any class of algebras. $(Q v \mathcal{K})_{\mathrm{RFSI}} \subseteq \mathrm{ISP}_{\mathrm{U}} \mathcal{K}$.

Proof. Let $\mathbf{A} \in(Q v K)_{\mathrm{RFSI}}$. Then $\mathbf{A} \in Q v K=\mathrm{ISPP}_{\mathrm{U}} \mathcal{K}$. Let $\mathbf{C}_{i} \in \mathrm{P}_{\mathrm{U}} \mathcal{K}$ with $i \in I$ be a system of algebras, and let $\mathbf{B}$ be a subalgebra of $\prod_{i \in I} \mathbf{C}_{i}$ such that $\mathbf{A} \cong \mathbf{B}$. Since $\mathbf{A}$ and hence $\mathbf{B}$ are relatively finitely subdirectly irreducible by hypothesis, $\Delta_{B}$ is finitely meet irreducible, and thus meet prime in $\operatorname{Con}_{Q v K} \mathbf{B}$. So by the lemma there is an ultrafilter $U$ on $I$ such that $\Psi_{J} \cap B^{2}=\Delta_{B}$ for every $J \in U$. Then the natural homomorphism of $\prod_{i \in I} \mathbf{C}_{i}$ onto $\prod_{i \in I} \mathbf{C}_{i} / U$ is one-one on the subalgebra $\mathbf{B}$. Thus $\mathbf{A} \in \operatorname{ISP}_{\mathrm{U}}\left(\mathrm{P}_{U} \mathcal{K}\right)=\operatorname{ISP}_{U} K$.

Relative subvarieties. Let $\mathcal{Q}$ be any quasivariety. A subquasivariety $R$ of $\mathcal{Q}$ is called a variety relative to $\mathcal{Q}$, or a relative subvariety of $\mathcal{Q}$, if $\mathcal{R}=\mathcal{V} \cap \mathcal{Q}$ for some variety $\mathcal{V}$.

If $\Gamma$ is a base for $\mathcal{Q}$, then every relative subvariety is of the form $\operatorname{Mod}(E \cup \Gamma)$ for some set of identities $E$.

LEMMA 1.4. Let $R$ be a relative subvariety of a quasivariety $\mathcal{Q}$.

(1) $\operatorname{Con}_{R} \mathbf{A}=\operatorname{Con}_{\mathcal{Q}} \mathbf{A}$ for every $\mathbf{A} \in R$;

(2) $R_{\mathrm{RFSI}}=R \cap \mathcal{Q}_{\mathrm{RFSI}}$;

(3) if $\mathcal{Q}$ is relatively congruence-distributive, then so is $R$.

Proof. (1) Clearly $\operatorname{Con}_{\mathcal{R}} \mathbf{A} \subseteq \operatorname{Con}_{\mathcal{Q}} \mathbf{A}$ since $R \subseteq \mathcal{Q}$. Assume $\Phi \in \operatorname{Con}_{\mathcal{Q}} \mathbf{A}$. Then $\mathbf{A} / \Phi \in \mathcal{Q}$, and $\mathbf{A} / \Phi \in \mathcal{V}$ since $\mathcal{V}$ is a variety. Thus $\mathbf{A} / \Phi \in \mathcal{V} \cap \mathcal{Q}=R$, and hence $\Phi \in \operatorname{Con}_{R} \mathbf{A}$.

(2) $\mathbf{A} \in R_{\mathrm{RFSI}}$ iff $\Delta_{A}$ is finitely meet irreducible in $\operatorname{Con}_{R} \mathbf{A}=\operatorname{Con}_{\mathcal{Q}} \mathbf{A}$ iff $\mathbf{A} \in \mathcal{Q}_{\text {RFSI }}$.

(3) Immediate from part (1). 
COROLlARY 1.5. Let $\mathcal{Q}$ be a quasivariety, and let $R$ and $S$ be relative subvarieties of $\mathcal{Q}$ such that $R \cap \mathcal{Q}_{\mathrm{RFSI}}=S \cap \mathcal{Q}_{\mathrm{RFSI}}$. Then $R=S$.

For any quasivariety $\mathcal{Q}$ and any $\mathcal{K} \subseteq \mathcal{Q}$ there is a smallest relative subvariety $R$ of $\mathcal{Q}$ such that $K \subseteq R$; we denote it by $V a_{\mathcal{Q}} K$. Note that $V a_{\mathcal{Q}} K=(V a K) \cap \mathcal{Q}$ where $V a K$ is the variety generated by $K$.

For any class $K$ of algebras, let $\mathrm{H}_{\mathcal{Q}} \mathcal{K}:=\mathrm{HK} \cap \mathcal{Q}$.

THEOREM 1.6. For any quasivariety $\mathcal{Q}$ and any $K \subseteq \mathcal{Q}$ we have $V a_{\mathcal{Q}} \mathcal{K}=$ $\mathrm{H}_{Q} \mathrm{SP} K$.

ProOF. $V a_{\mathcal{Q}} \mathcal{K}=(V a K) \cap \mathcal{Q}=($ HSP $K) \cap \mathcal{Q}=\mathrm{H}_{\mathcal{Q}}$ SP $\mathcal{Q}$.

The next theorem is the version of Jónsson's lemma for relative subvarieties. It was independently obtained by Dziobiak [15, Lemma 3.1].

THEOREM 1.7. Let $\mathcal{Q}$ be a relatively congruence-distributive quasivariety, and let $\mathrm{K} \subseteq \mathcal{Q}$. Then $\left(V a_{\mathcal{Q}} \mathrm{K}\right)_{\mathrm{RFSI}} \subseteq \mathrm{H}_{\mathcal{Q}} \mathrm{SP}_{\mathrm{U}} \mathrm{K}$.

Proof. Let $\mathbf{A} \in\left(V a_{\mathcal{Q}} \mathcal{K}\right)_{\mathrm{RFSI}}$. Then $\mathbf{A} \in V a_{\mathcal{Q}} \mathcal{K}=\mathrm{H}_{\mathcal{Q}} \mathrm{SP} K$. Thus there exist $\mathbf{C}_{i} \in \mathcal{K}$ for $i \in I, \mathbf{B} \subseteq \prod_{i \in I} \mathbf{C}_{i}$, and $\Phi \in C o n_{\mathcal{Q}} \mathbf{B}$ such that $\mathbf{A} \cong \mathbf{B} / \Phi$; moreover, since $\mathbf{A} \in \mathcal{Q}_{\mathrm{RFSI}}, \Phi$ is finitely meet irreducible in $\operatorname{Con}_{\mathcal{Q}} \mathbf{B}$, and hence meet prime since $\mathbf{C o n}_{\mathcal{Q}} \mathbf{B}$ is distributive by hypothesis. We now apply Lemma 1.2. There exists an ultrafilter $U$ on $I$ such that $\Psi_{J} \cap B^{2} \subseteq \Phi$ for each $J \in U$. Thus $\Phi$ includes the relation-kernel of the restriction to $\mathbf{B}$ of the natural homomorphism of $\prod_{i \in I} \mathbf{C}_{i}$ onto $\prod_{i \in I} \mathbf{C}_{i} / U$. So $\mathbf{B} / \Phi$ and hence $\mathbf{A}$ are $\mathcal{Q}$-homomorphic images of $\mathbf{B} / U \subseteq \prod_{i \in I} \mathbf{C}_{i} / U$.

2. Universally parameterized equationally definable principal congruence meets. In this section we show that, if a finitely generated quasivariety $\mathcal{Q}$ is relatively congruence-distributive, there exists a finite system of universally quantified equations that define the intersection of any pair of principal congruences of Q.

By a universally parameterized equation, or more simply a parameterized equation, we mean any formula of the form

$$
\forall \bar{u}(\kappa(\bar{x}, \bar{u}) \approx \lambda(\bar{x}, \bar{u}))
$$

where $\kappa(\bar{x}, \bar{u})$ and $\lambda(\bar{x}, \bar{u})$ are arbitrary terms with free variables $\bar{x}:=x_{0}, \ldots, x_{m-1}$ and parameters $\bar{u}:=u_{0}, \ldots, u_{n-1}$.

LEMMA 2.1. Let $\mathcal{Q}$ be a quasivariety and

$$
\forall \bar{u} \varphi(x, y, z, w, \bar{u}):=\forall \bar{u}\left(\bigwedge_{i<m} \tau_{i}(x, y, z, w, \bar{u}) \approx \sigma_{i}(x, y, z, w, \bar{u})\right)
$$

be a finite conjunction of parameterized equations. Then the following are equivalent:

(1) for all $\mathbf{A} \in \mathcal{Q}$ and all $a, b, c, d \in A$,

$$
\Theta_{\mathcal{Q}}(a, b) \cap \Theta_{\mathcal{Q}}(c, d)=\bigvee_{i<m} \bigvee_{\bar{e} \in A^{n}} \Theta_{\mathcal{Q}}\left(\tau_{i} \mathbf{A}(a, b, c, d, \bar{e}), \sigma_{i} \mathbf{A}(a, b, c, d, \bar{e})\right) \text {; }
$$

(2) for all $\mathbf{A} \in \mathcal{Q}$ and all $a, b, c, d \in A$,

$$
\Theta_{\mathcal{Q}}(a, b) \cap \Theta_{\mathcal{Q}}(c, d)=\Delta_{A} \Leftrightarrow \mathbf{A} \vDash \forall \bar{u} \varphi(a, b, c, d, \bar{u}) ;
$$

(3) $\mathcal{Q}_{\mathrm{RFSI}} \vDash \forall x y z w(\forall \bar{u} \varphi(x, y, z, w, \bar{u}) \leftrightarrow x \approx y \vee z \approx w)$. 
ProOF. The implications $(1) \Rightarrow(2) \Rightarrow(3)$ are immediate.

$(3) \Rightarrow(1)$. It follows immediately from (3) that $\tau_{i}(x, x, z, w, \bar{u}) \approx \sigma_{i}(x, x, z, w, \bar{u})$ is an identity of $\mathcal{Q}_{\mathrm{RFSI}}$, and hence of $\mathcal{Q}$, for each $i<m$. Let $\Phi:=\Theta_{\mathcal{Q}}(a, b)$. The quotient $\mathbf{A} / \Phi$ is contained in $\mathcal{Q}$ since $\Phi$ is a $\mathcal{Q}$-congruence by definition. Thus $\tau_{i}^{\mathbf{A}}(a, b, c, d, \bar{e}) / \Phi=\sigma_{i}^{\mathbf{A}}(a, b, c, d, \bar{e}) / \Phi$ for all $i<m$ and $\bar{e} \in A^{n}$, i.e.,

$$
\bigvee_{i<m} \bigvee_{\bar{e} \in A^{n}} \Theta_{\mathcal{Q}}\left(\tau_{i}^{\mathbf{A}}(a, b, c, d, \bar{e}), \sigma_{i}^{\mathbf{A}}(a, b, c, d, \bar{e})\right) \subseteq \Theta_{\mathcal{Q}}(a, b)
$$

By a similar argument the congruence on the left is also included in $\Theta_{\mathcal{Q}}(c, d)$. This establishes one of the two inclusions of part (1).

To get the other inclusion it suffices to show that, for each finitely meet irreducible $\Phi$ in $\operatorname{Con}_{\mathcal{Q}} \mathbf{A}$,

$$
\begin{array}{r}
\Phi \supseteq \bigvee_{i<m} \bigvee_{\bar{e} \in A^{n}} \Theta_{\mathcal{Q}}\left(\tau_{i}^{\mathbf{A}}(a, b, c, d, \bar{e}), \sigma_{i}^{\mathbf{A}}(a, b, c, d, \bar{e})\right) \\
\Rightarrow \Phi \supseteq \Theta_{\mathcal{Q}}(a, b) \cap \Theta_{\mathcal{Q}}(c, d) .
\end{array}
$$

Suppose the first inclusion holds. Then

$$
\tau_{i}^{\mathbf{A} / \Phi}(a / \Phi, b / \Phi, c / \Phi, d / \Phi, \bar{e} / \Phi)=\sigma_{i}^{\mathbf{A} / \Phi}(a / \Phi, b / \Phi, c / \Phi, d / \Phi, \bar{e} / \Phi)
$$

for all $i<m$ and all $\bar{e} \in A^{n}$. Thus $\mathbf{A} / \Phi \vDash \forall \bar{u} \varphi(a / \Phi, b / \Phi, c / \Phi, d / \Phi, \bar{u})$, and hence by part (3), $a / \Phi=b / \Phi$ or $c / \Phi=d / \Phi$, i.e., $\Phi \supseteq \Theta_{\mathcal{Q}}(a, b)$ or $\Phi \supseteq \Theta_{\mathcal{Q}}(c, d)$. Thus (2) holds.

A quasivariety has (universally) parameterized equationally definable relative principal meets (parameterized REDPM for short) if any (and hence all) of the conditions 2.1(1),(2),(3) hold for some conjunction of parameterized equations (1).

By an elementary class we mean any class of algebras definable by a possibly infinite set of sentences of first-order predicate logic.

THEOREM 2.2. A quasivariety $\mathcal{Q}$ has parameterized REDPM iff $\mathcal{Q}$ is relatively congruence-distributive and $\mathcal{Q}_{\mathrm{RFSI}}$ is an elementary class.

PrOOF. For the implication from left to right, assume the conjunction of parameterized equations (1) defines relative principal congruence meets in $\mathcal{Q}$. From 2.1(3) we at once get

$$
\mathcal{Q}_{\mathrm{RFSI}} \subseteq \operatorname{Mod}(\Gamma \cap\{\forall x y z w(\forall \bar{u} \varphi(x, y, z, w, \bar{u}) \rightarrow x \approx y \vee z \approx w)\}),
$$

where $\Gamma$ is any base for $\mathcal{Q}$. For the reverse inclusion, consider $\mathbf{A} \in \mathcal{Q}$ such that

$$
\mathbf{A} \vDash \forall x y z w(\forall \bar{u} \varphi(x, y, z, w, \bar{u}) \rightarrow x \approx y \vee z \approx w) .
$$

Using the fact $\forall \bar{u} \varphi(x, y, z, w, \bar{u})$ defines relative principal meets in $\mathbf{A}$, we get that $\Theta_{\mathcal{Q}}(a, b) \cap \Theta_{\mathcal{Q}}(c, d)=\Delta_{A}$ implies $\Theta_{\mathcal{Q}}(a, b)=\Delta_{A}$ or $\Theta_{\mathcal{Q}}(c, d)=\Delta_{A}$, for all $a, b, c, d \in A$, i.e., $\mathbf{A} \in \mathcal{Q}_{\mathrm{RFSI}}$.

So $\mathcal{Q}_{\mathrm{RFSI}}$ is elementary. To show $\mathcal{Q}$ is relatively congruence-distributive, it suffices to prove that 
for all $\mathbf{A} \in \mathcal{Q}$ and for all $\Phi, \Theta_{0}, \Theta_{1} \in \operatorname{Con}_{\mathcal{Q}} \mathbf{A}$. Since $\operatorname{Con}_{\mathcal{Q}} \mathbf{A}$ is algebraic, we can assume without loss of generality that $\Phi$ is principal and $\Theta_{0}, \Theta_{1}$ are finitely generated, i.e., it suffices to prove

$$
\Theta_{\mathcal{Q}}(a, b) \cap \bigvee_{j<k} \Theta_{\mathcal{Q}}\left(c_{j}, d_{j}\right) \subseteq \bigvee_{j<k} \Theta_{\mathcal{Q}}(a, b) \cap \Theta_{\mathcal{Q}}\left(c_{j}, d_{j}\right),
$$

for all $a, b, c_{0}, \ldots, c_{k-1}, d_{0}, \ldots, d_{k-1} \in A$. We prove this inclusion by showing that every finitely meet irreducible $\mathcal{Q}$-congruence that includes the right-hand side also includes the left-hand side. Let $\Psi$ be a finitely meet irreducible $\mathcal{Q}$-congruence such that $\Theta_{\mathcal{Q}}(a, b) \cap \Theta_{\mathcal{Q}}(c, d) \subseteq \Psi$ for all $j<k$. Then $\tau_{i}^{\mathbf{A}}\left(a, b, c_{j}, d_{j}, \bar{e}\right) \equiv$ $\sigma_{i}^{\mathbf{A}}\left(a, b, c_{j}, d_{j}, \bar{e}\right)(\bmod \Psi)$ for all $i<m, j<k$, and $\bar{e} \in A^{n}$ (see 2.1(1)). Therefore, $\mathbf{A} / \Psi \vDash \forall \bar{u} \varphi\left(a, b, c_{j}, d_{j}, \bar{u}\right)$ for all $j<k$. So either $a \equiv b(\bmod \Psi)$, or $c_{j} \equiv d_{j}(\bmod$ $\Psi)$ for all $j<k$, i.e., either $\Theta_{\mathcal{Q}}(a, b) \subseteq \Psi$ or $\bigvee_{j<k} \Theta_{\mathcal{Q}}\left(c_{j}, d_{j}\right) \subseteq \Psi$. Thus (3) holds, and $\mathcal{Q}$ is relatively congruence-distributive.

We now prove the implication from right to left. We assume $\mathcal{Q}$ is relatively congruence-distributive and $\mathcal{Q}_{\mathrm{RFSI}}$ is elementary. Let $\mathbf{F}$ be the free algebra of $\mathcal{Q}$ over the free generators $x, y, z, w, u_{0}, u_{1}, \ldots$ Let $\left\langle\tau_{i}(x, y, z, w, \bar{u}), \sigma_{i}(x, y, z, w, \bar{u})\right\rangle$, for $i<\omega$, be a system of generators for the intersection of the principal congruences generated by $\langle x, y\rangle$ and $\langle z, w\rangle$, i.e.,

$$
\Theta_{\mathcal{Q}} \mathbf{F}(x, y) \cap \Theta_{\mathcal{Q}} \mathbf{F}(z, w)=\bigvee_{i<\omega} \Theta_{\mathcal{Q}} \mathbf{F}\left(\tau_{i}(x, y, z, w, \bar{u}), \sigma_{i}(x, y, z, w, \bar{u})\right)
$$

(We identify terms in the language of $\mathcal{Q}$ with elements of the free algebra; there are only countably many because of the assumption that the language type is finite.) Let $\mathbf{A}$ be any countably generated algebra in $\mathcal{Q}$, and let $a, b, c, d \in A$; let $e_{0}, e_{1}, e_{2}, \ldots$ be any sequence of elements of $A$ that together with $a, b, c, d$ generate A. Let $h: \mathbf{F} \rightarrow \mathbf{A}$ be the surjective homomorphism such that $h x=a, h y=b$, $h z=c, h w=d$, and $h u_{i}=e_{i}$ for $i<\omega$. Let $\Phi \in C o n_{\mathcal{Q}} \mathbf{F}$ be the relation-kernel of $h$. Then for all $p, r \in A$ we have $h^{-1}\left[\Theta_{\mathcal{Q}} \mathbf{A}(p, r)\right]=\Theta_{\mathcal{Q}} \mathbf{F}(\pi, \rho) \vee \Phi$, where $\pi$ and $\rho$ are any pair of terms such that $h \pi=p$ and $h \rho=r$. Now making use of relative congruence-distributivity we have

$$
\begin{aligned}
h^{-1}\left[\Theta_{\mathcal{Q}} \mathbf{A}(a, b) \cap \Theta_{\mathcal{Q}} \mathbf{A}(c, d)\right] & =h^{-1}\left[\Theta_{\mathcal{Q}} \mathbf{A}(a, b)\right] \cap h^{-1}\left[\Theta_{\mathcal{Q}} \mathbf{A}(c, d)\right] \\
= & \left(\Theta_{\mathcal{Q}} \mathbf{F}(x, y) \vee \Phi\right) \cap\left(\Theta_{\mathcal{Q}} \mathbf{F}(z, w) \vee \Phi\right) \\
= & \left(\bigvee_{i<\omega} \Theta_{\mathcal{Q}}^{\mathbf{F}}\left(\tau_{i}(x, y, z, w, \bar{u}), \sigma_{i}(x, y, z, w, \bar{u})\right)\right) \vee \Phi \\
= & \bigvee_{i<\omega}\left(\Theta_{\mathcal{Q}}^{\mathbf{F}}\left(\tau_{i}(x, y, z, w, \bar{u}), \sigma_{i}(x, y, z, w, \bar{u})\right) \vee \Phi\right) \\
= & \bigvee_{i<\omega} h^{-1}\left[\Theta_{\mathcal{Q}}^{\mathbf{A}}\left(\tau_{i}^{\mathbf{A}}(a, b, c, d, \bar{e}), \sigma_{i}^{\mathbf{A}}(a, b, c, d, \bar{e})\right)\right] \\
= & h^{-1}\left[\bigvee_{i<\omega} \Theta_{\mathcal{Q}}^{\mathbf{A}}\left(\tau_{i}^{\mathbf{A}}(a, b, c, d, \bar{e}), \sigma_{i}^{\mathbf{A}}(a, b, c, d, \bar{e})\right)\right] .
\end{aligned}
$$

Thus

$$
\Theta_{\mathcal{Q}}^{\mathbf{A}}(a, b) \cap \Theta_{\mathcal{Q}}^{\mathbf{A}}(c, d)=\bigvee_{i<\omega} \Theta_{\mathcal{Q}}^{\mathbf{A}}\left(\tau_{i}^{\mathbf{A}}(a, b, c, d, \bar{e}), \sigma_{i}^{\mathbf{A}}(a, b, c, d, \bar{e})\right)
$$


Therefore, for every countably generated $\mathbf{A} \in \mathcal{Q}$ and all $a, b, c, d \in A$ we have

$$
\begin{aligned}
\Theta_{\mathcal{Q}}^{\mathbf{A}}(a, b) \cap \Theta_{\mathcal{Q}}^{\mathbf{A}}(c, d)= & \Delta_{A} \\
& \Leftrightarrow \tau_{i}^{\mathbf{A}}(a, b, c, d, \bar{e})=\sigma_{i}^{\mathbf{A}}(a, b, c, d, \bar{e}) \text { for all } i<\omega \text { and } \\
& \text { all } \bar{e} \in A^{n} \text { such that } a, b, c, d, e_{0}, e_{1}, \ldots \text { generate } \mathbf{A} .
\end{aligned}
$$

Observe first of all that the equivalence (4) continues to hold with the qualification "such that $a, b, c, d, e_{0}, e_{1}, \ldots$ generate $\mathbf{A}$ " removed. Because if $\Theta_{\mathcal{Q}}^{\mathbf{A}}(a, b) \cap$ $\Theta_{\mathcal{Q}}^{\mathbf{A}}(c, d)=\Delta_{A}$, then clearly $\Theta_{\mathcal{Q}}^{\mathbf{B}}(a, b) \cap \Theta_{\mathcal{Q}}^{\mathbf{B}}(c, d)=\Delta_{B}$ for any subalgebra $\mathbf{B}$ of $\mathbf{A}$, in particular the subalgebra generated by $a, b, c, d, e_{0}, e_{1}, \ldots$. Next observe that (4) holds for all $\mathbf{A} \in \mathcal{Q}$ regardless of the number of generators, because $\Theta_{\mathcal{Q}}^{\mathbf{A}}(a, b) \cap \Theta_{\mathcal{Q}}^{\mathbf{A}}(c, d)=\Delta_{A}$ iff $\Theta_{\mathcal{Q}}^{\mathbf{B}}(a, b) \cap \Theta_{\mathcal{Q}}^{\mathbf{B}}(c, d)=\Delta_{B}$ for every countably generated subalgebra $\mathbf{B}$ of $\mathbf{A}$ that contains $a, b, c, d$. Thus $\mathcal{Q}_{\mathrm{RFSI}}$ satisfies

$$
\forall x y z w\left(\bigwedge_{i<\omega} \forall \bar{u}\left(\tau_{i}(x, y, z, w, \bar{u}) \approx \sigma_{i}(x, y, z, w, \bar{u})\right) \leftrightarrow x \approx y \vee z \approx w\right) .
$$

Finally, using the assumption $\mathcal{Q}_{\mathrm{RFSI}}$ is elementary it is easy to show that $\mathcal{Q}_{\mathrm{RFSI}}$ continues to satisfy (5) when the infinite conjunction is replaced by a finite subconjunction

$$
\bigwedge_{i<m} \forall \bar{u}\left(\tau_{i}(x, y, z, w, \bar{u})=\sigma_{i}(x, y, z, w, \bar{u})\right) .
$$

Thus by 2.1(3), $\mathcal{Q}$ has parameterized REDPM.

COROLLARY 2.3. Every finitely generated and relatively congruence-distributive quasivariety has parameterized REDPM.

Proof. Assume $\mathcal{Q}=Q v \mathcal{K}$ where $\mathcal{K}$ is a finite set of finite algebras. In view of the theorem it will suffice to show that $\mathcal{Q}_{\mathrm{RFSI}}$ is included in IS $K$ and hence is finite. But by the relativized Jónsson lemma, Theorem $1.3, \mathcal{Q}_{\mathrm{RFSI}} \subseteq \operatorname{ISP}_{\mathrm{U}} \mathcal{K}=\mathrm{IS} \mathcal{K}$.

Metalogical analogues of Theorem 2.2 and its corollary were proved for protoalgebraic deductive systems in [9]. The two results in essentially their present form were independently established by J. Czelakowski and W. Dziobiak.

3. First main lemma. The purpose of this section is to prove Theorem 3.1 below. It differs from Theorem II (see the Introduction) at several points. The main difference is the additional premiss that the underlying quasivariety $\mathcal{Q}$, which includes $R$ as a relative subvariety, is finitely based. On the other hand, it assumes only that $R$ is relatively congruence-distributive, and not $\mathcal{Q}$. Also, instead of $R$ being finitely generated, it assumes only that $R_{\mathrm{RFSI}}$ is a finitely axiomatizable elementary class. 3.1 plays a key role in the proof of Theorem I. As mentioned in the Introduction, Theorem II itself is obtained as an immediate corollary of I. A class is strictly elementary if it is defined by a finite set of first-order sentences.

THEOREM 3.1. Assume $\mathcal{Q}$ is a finitely based quasivariety and $\mathcal{R}$ is a relative subvariety of $\mathcal{Q}$. If $R$ is relatively congruence-distributive and $R_{\mathrm{RFSI}}$ is strictly elementary, then $R$ is finitely based.

The theorem can be reformulated in syntactical terms as follows: Let $R$ be a quasivariety such that $R=\operatorname{Mod}(\Gamma \cup E)$, where $\Gamma$ is some finite set of quasi-identities and $E$ is a possibly infinite set of identities. If $R$ is relatively congruence-distributive and $R_{\mathrm{RFSI}}$ is strictly elementary, then there is a finite subset $E^{\prime}$ of $E$ such that $R=\operatorname{Mod}\left(\Gamma \cup E^{\prime}\right)$. 
COROLLARY 3.2. Let $\mathcal{Q}$ be a finitely based and relatively congruence-distributive quasivariety. Let $\mathcal{R}$ be any relative subvariety of $\mathcal{Q}$ such that $R_{\mathrm{RFSI}}$ is strictly elementary. Then $R$ is finitely based.

PROOF. $R$ is relatively congruence-distributive since it is a relative subvariety of a quasivariety with the property.

COROLLARY 3.3. Let $\mathcal{Q}$ be a finitely based and relatively congruence-distributive quasivariety. Then every finitely generated relative subvariety of $\mathcal{Q}$ is finitely based.

ProOF. Let $\mathcal{M} \subseteq \mathcal{Q}$ be a finite set of finite algebras. Then

$$
\left(V a_{\mathcal{Q}} \mathcal{M}\right)_{\mathrm{RFSI}} \subseteq \mathrm{H}_{\mathcal{Q}} \mathrm{SP}_{\mathrm{U}} \mathcal{M}=\mathrm{H}_{\mathcal{Q}} \mathrm{S} \mathcal{M} \text {. }
$$

Thus up to isomorphism $\left(\mathrm{Va}_{Q} \mathcal{M}\right)_{\mathrm{RFSI}}$ is a finite set of finite algebras.

The proof of the theorem will be given in two lemmas. Let $\mathcal{Q}=\operatorname{Mod} \Gamma$ with $\Gamma$ a finite set of quasi-identities. Let $R=\operatorname{Mod}(\Gamma \cup E)$ where $E$ is a possibly infinite set of identities. Let $\forall \bar{u} \varphi(x, y, z, w, \bar{u})$ be a conjunction of parameterized equations that defines principal $\mathcal{Q}$-congruence meets in $\mathcal{R}$. (Recall that $\mathcal{Q}$ - and $\mathcal{R}$-congruences coincide for members of $R$.) Then

$$
R_{\mathrm{RFSI}}=\operatorname{Mod}(\Gamma \cup E \cup\{\forall x y z w(\forall \bar{u} \varphi(x, y, z, w, \bar{u}) \rightarrow x \approx y \vee z \approx w)\}) .
$$

Since $R_{\mathrm{RFSI}}$ is strictly elementary, there exists a finite $E^{\prime} \subseteq E$ such that

$$
R_{\mathrm{RFSI}}=\operatorname{Mod}\left(\Gamma \cup E^{\prime} \cup\{\forall x y z w(\forall \bar{u} \varphi(x, y, z, w, \bar{u}) \rightarrow x \approx y \vee z \approx w)\}\right) .
$$

Let $\Omega$ be the set consisting of the following formulas:

$$
\begin{gathered}
\forall \bar{u} \varphi(x, x, z, w, \bar{u}) ; \\
\forall \bar{u} \varphi(x, y, z, w, \bar{u}) \rightarrow \forall \bar{u} \varphi(y, x, z, w, \bar{u}) ; \\
\forall \bar{u} \varphi(x, y, z, w, \bar{u}) \wedge \forall \bar{u} \varphi(y, v, z, w, \bar{u}) \rightarrow \forall \bar{u} \varphi(x, v, z, w, \bar{u}) ;
\end{gathered}
$$

for each operation symbol $\omega$,

$$
\left(\bigwedge_{i<m} \forall \bar{u} \varphi\left(x_{i}, y_{i}, z, w, \bar{u}\right)\right) \rightarrow \forall \bar{u} \varphi\left(\omega x_{0} \cdots x_{m-1}, \omega y_{0} \cdots y_{m-1}, z, w, \bar{u}\right) ;
$$

for each quasi-equation $\psi(\bar{x}):=\left(\bigwedge_{i<k} \kappa_{i}(\bar{x}) \approx \lambda_{i}(\bar{x})\right) \rightarrow \pi(\bar{x}) \approx \rho(\bar{x})$ in $\Gamma$,

$$
\left(\bigwedge_{i<k} \forall \bar{u} \varphi\left(\kappa_{i}(\bar{x}), \lambda_{i}(\bar{x}), z, w, \bar{u}\right)\right) \rightarrow \forall \bar{u} \varphi(\pi(\bar{x}), \rho(\bar{x}), z, w, \bar{u}) ;
$$

and finally,

$$
\forall \bar{u} \varphi(x, y, z, w, \bar{u}) \rightarrow \forall \bar{u} \varphi(z, w, x, y, \bar{u})
$$

$$
\forall \bar{u} \varphi(x, y, x, y, \bar{u}) \rightarrow x \approx y .
$$

LEMMA 3.4. $R \vDash \Omega$, i.e., $\Gamma \cup E \vDash \Omega$.

ProOF. Let $\mathbf{A} \in R$. Since $\forall \bar{u} \varphi$ defines relative principal congruence meets in $R$, each formula of $\Omega$ is equivalent to a corresponding congruence formula. For instance, (1) is equivalent to $\Theta_{\mathcal{Q}}(a, a) \cap \Theta_{\mathcal{Q}}(c, d)=\Delta_{A}$, and (7) is equivalent to

$$
\Theta_{\mathcal{Q}}(a, b) \cap \Theta_{\mathcal{Q}}(a, b)=\Delta_{A} \Rightarrow a=b .
$$


Similarly, (2), (4), and the (6) are equivalent to congruence formulas that hold trivially. The congruence conditions corresponding to (3) and (5) also hold, but they depend on the distributivity of $\operatorname{Con}_{R} \mathbf{A}$. Consider the condition corresponding to (5) for example: for each quasi-equation $\psi(\bar{x}):=\left(\bigwedge_{i<k} \kappa_{i}(\bar{x}) \approx \lambda_{i}(\bar{x})\right) \rightarrow$ $\pi(\bar{x}) \approx \rho(\bar{x})$ in $\Gamma$

$$
\begin{aligned}
\Theta_{\mathcal{Q}}\left(\kappa_{i}^{\mathbf{A}}(\bar{a}), \lambda_{i}^{\mathbf{A}}(\bar{a})\right) \cap \Theta_{\mathcal{Q}}(c, d)=\Delta_{A} \quad \text { for all } i<m \\
\quad \Rightarrow \Theta_{\mathcal{Q}}\left(\pi^{\mathbf{A}}(\bar{a}), \rho^{\mathbf{A}}(\bar{a})\right) \cap \Theta_{\mathcal{Q}}(c, d)=\Delta_{A} .
\end{aligned}
$$

Since $\psi(\bar{x})$ is a quasi-identity of $R$, we have

$$
\Theta_{\mathcal{Q}}\left(\pi^{\mathbf{A}}(\bar{a}), \rho^{\mathbf{A}}(\bar{a})\right) \subseteq \bigvee_{i<k} \Theta_{\mathcal{Q}}\left(\kappa_{i}^{\mathbf{A}}(\bar{a}), \lambda_{i}^{\mathbf{A}}(\bar{a})\right)
$$

where the join on the right is in the lattice of $\mathcal{Q}$-congruences. Thus

$$
\Theta_{\mathcal{Q}}(c, d) \cap \Theta_{\mathcal{Q}}\left(\pi^{\mathbf{A}}(\bar{a}), \rho^{\mathbf{A}}(\bar{a})\right) \subseteq \bigvee_{i<k} \Theta_{\mathcal{Q}}(c, d) \cap \Theta_{\mathcal{Q}}\left(\kappa_{i}^{\mathbf{A}}(\bar{a}), \lambda_{i}^{\mathbf{A}}(\bar{a})\right)
$$

Let

$$
\mathcal{K}:=\operatorname{Mod}\left(\Gamma \cup E^{\prime} \cup \Omega\right)
$$

$K$ is not necessarily a quasivariety, but it is finitely axiomatizable since each of $\Gamma$, $E^{\prime}$, and $\Omega$ is finite.

LEMMA 3.5. $K \cap \mathcal{Q}_{\mathrm{RFSI}} \subseteq R_{\mathrm{RFSI}}$.

ProOF. Let $\mathbf{A} \in \mathcal{K} \cap \mathcal{Q}_{\mathrm{RFSI}}$. Since $\mathbf{A} \vDash \Gamma \cup E^{\prime}$, to show $\mathbf{A} \in R_{\mathrm{RFSI}}$ it suffices to show that

$$
\mathbf{A} \vDash \forall x y z w(\forall \bar{u} \varphi(x, y, z, w, \bar{u}) \rightarrow x \approx y \vee z \approx w)
$$

Consider $a, b, c, d \in A$ such that $\mathbf{A} \vDash \forall \bar{u} \varphi(a, b, c, d, \bar{u})$. Let $\Phi$ be the binary relation defined in $\mathbf{A}$ by $\forall \bar{u} \varphi(x, y, c, d, \bar{u})$, i.e.,

$$
\Phi:=\{\langle e, f\rangle: \mathbf{A} \vDash \forall \bar{u} \varphi(e, f, c, d, \bar{u})\} .
$$

$\Phi$ is a congruence because (1)-(4) are universally satisfied in $\mathbf{A}$, and it is a $\mathcal{Q}$-congruence by (5). By assumption $\langle a, b\rangle \in \Phi$.

Let

$$
\boldsymbol{\Psi}:=\{\langle g, h\rangle: \mathbf{A} \vDash \forall \bar{u} \varphi(e, f, g, h, \bar{u}) \text { for all }\langle e, f\rangle \in \Phi\} .
$$

Because of (6) $\Psi$ is also a $\mathcal{Q}$-congruence, and $\langle c, d\rangle \in \Psi$. Suppose $\langle e, f\rangle \in \Phi \cap \Psi$. Then $\mathbf{A} \vDash \forall \bar{u} \varphi(e, f, e, f, \bar{u})$, and hence we must have $e=f$ by (8). So $\Phi \cap \Psi=\Delta_{A}$, which implies $\Phi=\Delta_{A}$ or $\Psi=\Delta_{A}$ since $\mathbf{A} \in \mathcal{Q}_{\mathrm{RFSI}}$. Thus $a=b$ or $c=d$, and so (8) holds.

Since $\Gamma \cup E \vDash \Gamma \cup E^{\prime} \cup \Omega$ (Lemma 3.4), there exists a finite set $E^{\prime \prime}$ of identities such that $E^{\prime} \subseteq E^{\prime \prime} \subseteq E$ and $\Gamma \cup E^{\prime \prime} \vDash \Gamma \cup E^{\prime} \cup \Omega$. Therefore,

$$
R \subseteq \operatorname{Mod}\left(\Gamma \cup E^{\prime \prime}\right) \subseteq \mathcal{K} \subseteq \mathcal{Q},
$$

and hence $R \cap \mathcal{Q}_{\mathrm{RFSI}} \subseteq \operatorname{Mod}\left(\Gamma \cup E^{\prime \prime}\right) \cap \mathcal{Q}_{\mathrm{RFSI}}$. But by the last lemma the inclusion in the reverse direction also holds. (Recall that since $R$ is a relative subvariety, $R_{\mathrm{RFSI}}=R \cap \mathcal{Q}_{\mathrm{RFSI}}$. $)$ Thus $R=\operatorname{Mod}\left(\Gamma \cup E^{\prime \prime}\right)$ by Corollary 1.5 , since $\operatorname{Mod}\left(\Gamma \cup E^{\prime \prime}\right)$ is also a relative subvariety of $\mathcal{Q}$. This completes the proof of the theorem. 
4. Universally parameterized quasi-equations. The proof of Theorem I, which will be given in $\S 6$, is similar to that of Theorem 3.1 but more complicated in details. The key to the proof of 3.1 is the existence of the strictly elementary subclass $\mathcal{K}$ of $\mathcal{Q}$ whose finitely subdirectly irreducibles (relative to $\mathcal{Q}$ ) coincide with those of $R$. The key in turn to the construction of $\mathcal{K}$ is the fact that the definability of principal $\mathcal{Q}$-congruence meets, by the formula $\forall \bar{u} \varphi(x, y, z, w, \bar{u})$, turns out to be a finitely axiomatizable property of algebras. More precisely, there exists a finitely axiomatizable extension of $R$, viz. $K$, with the property that principal Q-congruence meets in $K$ are definable by $\forall \bar{u} \varphi(x, y, z, w, \bar{u}) .{ }^{1}$ But the existence of $\mathcal{K}$ depends essentially on the assumption that $\mathcal{Q}$ is finitely based. We run into difficulties when we attempt to extend the proof of 3.1 to the case $R$ is not a relative subvariety of a finitely based quasivariety. It turns out however that we can define in this case an extension $\mathcal{L}$ of $R$ that like $K$ is finitely axiomatizable, but in which the meet of principal congruences relative to the class $\mathcal{L}$ itself, rather than the larger class $\mathcal{Q}$, is definable. This requires an extension of the theory of congruences relative to a quasivariety to congruences relative to a model class defined by a more general class of formulas than quasi-identities; this is done in the present section. The next section contains the technical lemmas necessary for defining $\mathcal{L}$ and establishing its essential properties. The definition of $\mathcal{L}$ and the proofs of Theorems I and II are given in $\S 6$.

By a (universally) parameterized quasi-equation we mean any formula of the form

$$
\xi_{0}(\bar{x}) \wedge \cdots \wedge \xi_{k-1}(\bar{x}) \rightarrow \eta_{0}(\bar{x}) \wedge \cdots \wedge \eta_{l-1}(\bar{x})
$$

where the conjuncts $\xi_{i}(\bar{x})$ and $\eta_{j}(\bar{x})$ are arbitrary parameterized equations with free variables $\bar{x}:=x_{0}, x_{1}, \ldots, x_{p-1}$; by inserting dummy variables where necessary we can assume without loss of generality that the conjuncts all have the same system of universally quantified parameters, as well as the same free variables. Thus every parameterized quasi-equation is logically equivalent to a formula $\psi(\bar{x})$ of the form

$$
\forall \bar{v}\left(\bigwedge_{i<k} \kappa_{i}(\bar{x}, \bar{v}) \approx \lambda_{i}(\bar{x}, \bar{v})\right) \rightarrow \forall \bar{v}\left(\bigwedge_{j<l} \pi_{j}(\bar{x}, \bar{v}) \approx \rho_{j}(\bar{x}, \bar{v})\right),
$$

where $\bar{x}:=x_{0}, \ldots, x_{p-1}$ and $\bar{v}:=v_{0}, \ldots, v_{q-1}$. In the sequel $\psi(\bar{x})$ will always represent a parameterized quasi-equation and $\vartheta(\bar{x}), \xi(\bar{x}), \eta(\bar{x})$ arbitrary parameterized equations or conjunctions of parameterized equations. Every ordinary quasi-equation is a parameterized quasi-equation with an empty list of parameters. The formulas (1)-(7) that constitute the set $\Omega$ of the previous section are also examples of parameterized quasi-equations.

A class $\mathcal{L}$ of algebras is called a parameterized quasivariety if it is of the form $\mathcal{L}:=\operatorname{Mod} \Gamma$ where $\Gamma$ is a set of parameterized quasi-equations (more precisely a set of parameterized quasi-identities, i.e., universal closures of parameterized quasiequations).

An arbitrary binary relation $R$ on an algebra $\mathbf{A}$ is closed under the parameterized quasi-equation (1) if

$$
\left\{\left\langle\kappa_{i}^{\mathbf{A}}(\bar{a}, \bar{e}), \lambda_{i}^{\mathbf{A}}(\bar{a}, \bar{e})\right\rangle: i<k, \bar{e} \in A^{q}\right\} \subseteq R
$$

\footnotetext{
${ }^{1}$ Actually the situation is more complicated than described here, but it is useful to think in this way in trying to understand the structure of the proof of 3.1 and its relationship with the proof of Theorem I.
} 
always implies

$$
\left\{\left\langle\pi_{j}^{\mathbf{A}}(\bar{a}, \bar{e}), \rho_{j}^{\mathbf{A}}(\bar{a}, \bar{e})\right\rangle: j<l, \bar{e} \in A^{q}\right\} \subseteq R .
$$

If $\Phi \in$ Con $\mathbf{A}$, then $\mathbf{A} / \Phi \vDash \psi(\bar{x})$ iff $\Phi$ is closed under $\psi(\bar{x})$. Clearly, if each member of some family of congruences is closed under a given parameterized quasi-equation, then so is their intersection.

Let $\mathcal{L}$ be a parameterized quasivariety and let $\mathbf{A} \in \mathcal{L}$. Just as in the case of ordinary quasivarieties we call a congruence $\Phi$ on $\mathbf{A}$ an $\mathcal{L}$-congruence if $\mathbf{A} / \Phi \in \mathcal{L}$. $\Phi$ is a $\mathcal{L}$-congruence iff it is closed under any set of parameterized quasi-equations that forms a base for $\mathcal{L}$. So the set of all $\mathcal{L}$-congruences of $\mathbf{A}$. which we again denote by $\operatorname{Con}_{\mathcal{L}} \mathbf{A}$, is closed under arbitrary intersection, and thus forms a complete lattice $\operatorname{Con}_{\mathcal{L}} \mathbf{A}$. The fact that $\operatorname{Con}_{\mathcal{L}} \mathbf{A}$ is closed under intersection immediately gives

LEMMA 4.1. Every parameterized quasivariety is closed under the formation of subdirect products.

$\operatorname{Con}_{\mathcal{L}} \mathbf{A}$ need not be closed under the formation of unions of directed sets, so $\operatorname{Con}_{\mathcal{L}} \mathbf{A}$ is not in general an algebraic lattice. Thus the analogue of Birkhoff's subdirect product representation theorem cannot be expected to hold as it does for quasivarieties. However a restricted version sufficient for our purposes does hold.

For any parameterized quasivariety $\mathcal{L}$ the notion of an algebra $\mathbf{A} \in \mathcal{L}$ being finitely subdirectly irreducible relative to $\mathcal{L}$ is defined in the obvious way; the subclass of $\mathcal{L}$ so defined is denoted by $\mathcal{L}_{\text {RFSI }} . \quad \mathbf{A} \in \mathcal{L}$ is relatively finitely subdirectly irreducible iff $\Delta_{A}$ is finitely meet irreducible in the lattice $\operatorname{Con}_{\mathcal{L}} \mathbf{A}$. More generally, for any $\mathcal{L}$-congruence $\Phi, \mathbf{A} / \Phi \in \mathcal{L}_{\mathrm{RFSI}}$ iff $\Phi$ is finitely meet irreducible in $\operatorname{Con}_{\mathcal{L}} \mathbf{A}$.

$\operatorname{Con}_{\mathcal{L}} \mathbf{A}$ need not be algebraic if $\mathbf{A}$ is infinite, so a fixed $\mathcal{L}$-congruence, in particular $\Delta_{A}$, need not be the meet of finitely meet irreducible $\mathcal{L}$-congruences. This is always the case of course when $\mathbf{A}$ is finite, so we have

LEMMA 4.2. Assume $\mathcal{L}$ is a parameterized quasivariety, and let $\mathbf{A} \in \mathcal{L}$. If $\mathbf{A}$ is finite, then $\mathbf{A} \subseteq \mathrm{SD} \mathbf{C}_{0} \times \mathbf{C} \times \cdots \times \mathbf{C}_{n-1}$ for some $\mathbf{C}_{0}, \ldots, \mathbf{C}_{n-1} \in \mathcal{L}_{\mathrm{RFSI}}$.

5. The calculus of transformations. We deal almost exclusively with syntax in this section. The key notion is that of a transformation of a parameterized quasi-equation by a parameterized equation; this always generates another parameterized quasi-equation. We study the relationship between the model-theoretic properties of an arbitrary parameterized quasi-equation and those of its transform. The definition of transformation is given in three steps.

Let

$$
\forall \bar{u} \varphi(x, y, z, w, \bar{u}):=\forall \bar{u}\left(\bigwedge_{i<m} \tau_{i}(x, y, z, w, \bar{u}) \approx \sigma_{i}(x, y, z, w, \bar{u})\right),
$$

with $\bar{u}:=u_{0}, \ldots, u_{n-1}$, be a fixed but arbitrary conjunction of parameterized equations. For any pair of ordinary equations $\delta(\bar{x}):=\kappa(\bar{x}) \approx \lambda(\bar{x})$ and $\varepsilon(\bar{x}):=$ $\pi(\bar{x}) \approx \rho(\bar{x})$ define

$$
\operatorname{Tr}(\delta(\bar{x}), \varepsilon(\bar{x})):=\forall \bar{u} \varphi(\kappa(\bar{x}), \lambda(\bar{x}), \pi(\bar{x}), \rho(\bar{x}), \bar{u}) .
$$

Next, for any pair of conjunctions of parameterized equations

$$
\xi(\bar{x}):=\forall \bar{v}\left(\bigwedge_{i<k} \kappa_{i}(\bar{x}, \bar{v}) \approx \lambda_{i}(\bar{x}, \bar{v})\right), \eta(\bar{z}):=\forall \bar{v}\left(\bigwedge_{j<l} \pi_{i}(\bar{z}, \bar{v}) \approx \rho_{i}(\bar{z}, \bar{v})\right),
$$


define

$$
\operatorname{Tr}_{\varphi}(\xi(\bar{x}), \eta(\bar{z})):=\forall \bar{v}\left(\bigwedge_{i<k} \bigwedge_{j<l} \operatorname{Tr}_{\varphi}\left(\kappa_{i}(\bar{x}, \bar{v}) \approx \lambda_{i}(\bar{x}, \bar{v}), \pi_{j}(\bar{z}, \bar{v}) \approx \rho_{j}(\bar{z}, \bar{v})\right)\right) .
$$

Finally, for any parameterized quasi-equation $\psi(\bar{x})$

$$
\psi(\bar{x}):=\xi(\bar{x}) \rightarrow \eta(\bar{x}),
$$

where $\xi(\bar{x})$ and $\eta(\bar{x})$ are both conjunctions of parameterized equations, and any conjunction of parameterized equations $\vartheta(\bar{z})$, define

$$
\begin{aligned}
& \operatorname{Tr}_{\varphi}(\psi(\bar{x}), \vartheta(\bar{z})):=\operatorname{Tr}_{\varphi}(\xi(\bar{x}), \vartheta(\bar{z})) \rightarrow \operatorname{Tr}_{\varphi}(\eta(\bar{x}), \vartheta(\bar{z})), \\
& \operatorname{Tr}_{\varphi}(\vartheta(\bar{z}), \psi(\bar{x})):=\operatorname{Tr}_{\varphi}(\vartheta(\bar{z}), \xi(\bar{x})) \rightarrow \operatorname{Tr}_{\varphi}(\vartheta(\bar{z}), \eta(\bar{x})) .
\end{aligned}
$$

$\operatorname{Tr}_{\varphi}(\psi(\bar{x}), \vartheta(\bar{z}))$ and $\operatorname{Tr}_{\varphi}(\vartheta(\bar{z}), \psi(\bar{x}))$ are called respectively the left and right $\varphi$ transforms of $\psi(\bar{x})$ by $\vartheta(\bar{z})$.

Note that we define the transforms only of a parameterized quasi-equation by a conjunction of parameterized equations, not by another parameterized quasiequation. Note also that the transforms are again parameterized quasi-equations, so it makes sense to iterate the operation.

For any quasi-equation $\psi(\bar{x})$ define

$$
L t_{\varphi}(\psi(\bar{x})):=\operatorname{Tr}_{\varphi}(\psi(\bar{x}), z \approx w), \quad R t_{\varphi}(\psi(\bar{x})):=\operatorname{Tr}_{\varphi}(z \approx w, \psi(\bar{x})),
$$

where $z$ and $w$ are distinct variables not occurring among the $\bar{x}$ or among the bound variables $\bar{u}$ of $\forall \bar{u} \varphi$. L Lt $\psi$ $\psi$ and $R t_{\varphi} \psi$ are called simply the left and right $\varphi$-transforms of $\psi$, respectively.

Observe that the formula (1) of $\S 3$ is just the left transform of the trivial equation $x \approx x$. Similarly (2)-(4) are the left transforms of the quasi-equations $x \approx y \rightarrow y \approx$ $x, x \approx y \wedge y \approx z \rightarrow x \approx z$, and $\left(\bigwedge_{i<m} x_{i} \approx y_{i}\right) \rightarrow \omega x_{0} \cdots x_{m-1} \approx \omega y_{0} \cdots y_{m-1}$. Finally, (5) is the left transform of an arbitrary member of the base $\Gamma$ of $\mathcal{Q}$. In the proof of Lemma 3.5 we were able to conclude that the binary relation $\Phi$ was closed under all these quasi-identities, and thus formed a $\mathcal{Q}$-congruence, from the fact the algebra $\mathbf{A}$ universally satisfied the corresponding left transforms. Similarly, $\Psi$ was closed under the same quasi-identities because $\mathbf{A}$ satisfied the corresponding right transforms. This relationship between closure under a quasi-equation and satisfaction of its transforms extends to parameterized quasi-equations.

LEMMA 5.1. Let $\mathbf{A}$ be any algebra and $\forall \bar{u} \varphi(x, y, z, w, \bar{u})$ a conjunction of parameterized equations. Let $X \subseteq A \times A$ be any binary relation on $A$. Finally let

$$
\begin{aligned}
& \Phi_{X}:=\{\langle a, b\rangle \in A \times A: \mathbf{A} \vDash \forall \bar{u} \varphi(a, b, c, d, \bar{u}) \text { for all }\langle c, d\rangle \in X\}, \\
& \Psi_{X}:=\{\langle c, d\rangle \in A \times A: \mathbf{A} \vDash \forall \bar{u} \varphi(a, b, c, d, \bar{u}) \text { for all }\langle a, b\rangle \in X\} .
\end{aligned}
$$

Then for any parameterized quasi-equation $\psi$ we have

(1) $\mathbf{A} \vDash L t_{\varphi} \psi \Rightarrow \Phi_{X}$ is closed under $\psi$,

(2) $\mathbf{A} \vDash R t_{\varphi} \psi \Rightarrow \Psi_{X}$ is closed under $\psi$.

Proof. Let $\psi(\bar{x}):=\xi(\bar{x}) \rightarrow \eta(\bar{x})$, where

$$
\xi(\bar{x}):=\forall \bar{v}\left(\bigwedge_{i<k} \kappa_{i}(\bar{x}, \bar{v}) \approx \lambda_{i}(\bar{x}, \bar{v})\right)
$$




$$
\eta(\bar{x}):=\forall \bar{v}\left(\bigwedge_{j<l} \pi_{j}(\bar{x}, \bar{v}) \approx \rho_{j}(\bar{x}, \bar{v})\right)
$$

and where $\bar{x}:=x_{0}, \ldots, x_{p-1}$ and $\bar{v}:=v_{0}, \ldots, v_{q-1}$. Expanding the various definitions we see that $L t_{\varphi}(\psi(\bar{x}))$ has the form

$$
\begin{aligned}
\forall \bar{v}\left(\bigwedge_{i<k} \forall \bar{u} \varphi\left(\kappa_{i}(\bar{x}, \bar{v}), \lambda_{i}(\bar{x}, \bar{v}), z, w, \bar{u}\right)\right) & \\
& \rightarrow \forall \bar{v}\left(\bigwedge_{j<l} \forall \bar{u} \varphi\left(\pi_{j}(\bar{x}, \bar{v}), \rho_{j}(\bar{x}, \bar{v}), z, w, \bar{u}\right)\right) .
\end{aligned}
$$

Assume this parameterized quasi-equation is universally satisfied in $\mathbf{A}$. To show $\Phi_{X}$ is closed under $\psi$ consider any $\bar{a} \in A^{p}$, and suppose $\left\langle\kappa_{i}^{\mathbf{A}}(\bar{a}, \bar{e}), \lambda_{i}^{\mathbf{A}}(\bar{a}, \bar{e})\right\rangle \in \Phi_{X}$ for all $i<k$ and $\bar{e} \in A^{q}$. Then by definition of $\Phi_{X}$,

$$
\mathbf{A} \vDash \forall \bar{u} \varphi\left(\kappa_{i}^{\mathbf{A}}(\bar{a}, \bar{e}), \lambda_{i}^{\mathbf{A}}(\bar{a}, \bar{e}), c, d, \bar{u}\right)
$$

for all $i\left\langle k, \bar{e} \in A^{q}\right.$, and $\langle c, d\rangle \in X$. But by assumption $\mathbf{A}$ universally satisfies $L t_{\varphi}(\psi)$. Thus $\mathbf{A} \vDash \forall \bar{u} \varphi\left(\pi_{j}^{\mathbf{A}}(\bar{a}, \bar{e}), \rho_{j}^{\mathbf{A}}(\bar{a}, \bar{e}), c, d, \bar{u}\right)$ for all $j<k, \bar{e} \in A^{q}$, and $\langle c, d\rangle \in X$, i.e., $\left\langle\pi_{j}^{\mathbf{A}}(\bar{a}, \bar{e}), \rho_{j}^{\mathbf{A}}(\bar{a}, \bar{e})\right\rangle \in \Phi_{X}$ for all $j<l$ and $\bar{e} \in A^{q}$. So $\Phi_{X}$ is closed under $\psi$.

This gives the implication of part (1); part (2) is obtained the same way.

Let $\mathcal{Q}$ be a quasivariety in which $\forall \bar{u} \varphi$ defines relative principal congruence meets. The real content of the proof of Lemma 3.4 is that, whenever $\mathcal{Q}$ satisfies a quasiequation $\psi$ identically, it also satisfies its left and right $\varphi$-transform. This extends to parameterized quasi-equations.

LEMMA 5.2. Let $\mathcal{Q}$ be a quasivariety with parameterized REDPM, and assume that principal $\mathcal{Q}$-congruence meets are defined by a conjunction of parameterized equations $\forall \bar{u} \varphi(x, y, z, w, \bar{u})$. Then

$$
\mathcal{Q} \vDash L t_{\varphi} \psi \quad \text { and } \quad \mathcal{Q} \vDash R t_{\varphi} \psi,
$$

for every parameterized quasi-equation $\psi$ such that $\mathcal{Q} \vDash \psi$.

PROOF. Since every algebra in $\mathcal{Q}$ is a subdirect product of members of $\mathcal{Q}_{\mathrm{RFSI}}$, and parameterized quasi-equations are preserved by subdirect products, it suffices to prove that $\mathcal{Q}_{\mathrm{RFSI}} \vDash L t_{\varphi} \psi$ (a similar proof works for the right transform).

Suppose $\psi(\bar{x}):=\xi(\bar{x}) \rightarrow \eta(\bar{x})$ where $\xi(\bar{x})$ and $\eta(\bar{x})$ are as in (2) and (3). Then $L t_{\varphi}(\psi(\bar{x}))$ is given in (4). Let $\mathbf{A} \in \mathcal{Q}_{\mathrm{RFSI}}$, and assume $\bar{a} \in A^{p}$ and $c, d \in A$ satisfy the antecedent of $\left(L t_{\varphi} \psi\right)(\bar{x}, z, w)$, i.e.,

$$
\mathbf{A} \vDash \forall \bar{u} \varphi\left(\kappa_{i}^{\mathbf{A}}(\bar{a}, \bar{e}), \lambda_{i}^{\mathbf{A}}(\bar{a}, \bar{e}), c, d, \bar{u}\right) \quad \text { for all } i<k \text { and } \bar{e} \in A^{q} .
$$

Because $\forall \bar{u} \varphi$ defines relative principal meets by assumption,

$$
\Theta_{\mathcal{Q}}\left(\kappa_{i}^{\mathbf{A}}(\bar{a}, \bar{e}), \lambda_{i}^{\mathbf{A}}(\bar{a}, \bar{e})\right) \cap \Theta_{\mathcal{Q}}(c, d)=\Delta_{A}
$$

for all $i<k$ and $\bar{e} \in A^{q}$. Thus, since $\mathbf{A}$ is relatively finitely subdirectly irreducible, either $\kappa_{i}^{\mathbf{A}}(\bar{a}, \bar{e})=\lambda_{i}^{\mathbf{A}}(\bar{a}, \bar{e})$ for all $i<k$ and $\bar{e} \in A^{q}$, or $c=d$. In the first case $\bar{a}$ satisfies the antecedent of $\psi(\bar{x})$ in $\mathbf{A}$, and hence by hypothesis also the consequent, 
i.e., $\pi_{j}^{\mathbf{A}}(\bar{a}, \bar{e})=\rho_{j}^{\mathbf{A}}(\bar{a}, \bar{e})$ for all $j<l$ and $\bar{e} \in A^{q}$. So $\Theta_{\mathcal{Q}}\left(\pi_{j}^{\mathbf{A}}(\bar{a}, \bar{e}), \rho_{j}^{\mathbf{A}}(\bar{a}, \bar{e})\right) \cap$ $\Theta_{\mathcal{Q}}(c, d)=\Delta_{A}$, for all $j<l$ and $\bar{e} \in A^{q}$. We obtain the same conclusion in the second case, i.e., when $c=d$. Thus in both cases we have

$$
\mathbf{A} \vDash \forall \bar{u} \varphi\left(\pi_{j}^{\mathbf{A}}(\bar{a}, \bar{e}), \rho_{j}^{\mathbf{A}}(\bar{a}, \bar{e}), c, d, \bar{u}\right) \quad \text { for all } j<l \text { and } \bar{e} \in A^{q},
$$

and so $\bar{a}, c$, and $d$ must satisfy the consequent of $L t_{\varphi}(\psi)$.

In order to axiomatize the property of defining relative principal congruence meets we consider the following two formulas:

$$
\alpha:=\operatorname{Tr}_{\varphi}(x \approx y, z \approx w) \rightarrow \operatorname{Tr}_{\varphi}(z \approx w, x \approx y),
$$

(6) $\quad \beta:=\operatorname{Tr}_{\varphi}\left(\operatorname{Tr}_{\varphi}(x \approx y, z \approx w), r \approx s\right) \leftrightarrow \operatorname{Tr}_{\varphi}\left(x \approx y, \operatorname{Tr}_{\varphi}(z \approx w, r \approx s)\right)$,

where $x, y, z, w, r$, and $s$ are distinct variables. Note that $\alpha$ is a parameterized quasi-equation and $\beta$ is the conjunction of two parameterized quasi-equations.

LEMMA 5.3. Assume $\mathcal{Q}$ is a quasivariety with parameterized REDPM, and let $\forall \bar{u} \varphi(x, y, z, w, \bar{u})$ be a conjunction of parameterized equations that defines principal $\mathcal{Q}$-congruence meets. Then $\mathcal{Q} \vDash \alpha$ and $\mathcal{Q} \vDash \beta$.

PROOF. Since $\alpha$ and $\beta$ are parameterized quasi-equations, and hence preserved under the formation of subdirect products, it suffices to show that $\mathcal{Q}_{\mathrm{RFSI}} \vDash \alpha$ and $\mathcal{Q}_{\text {RFSI }} \vDash \beta$.

To show that $\mathcal{Q}_{\mathrm{RFSI}} \vDash \beta$, let $\mathbf{A} \in \mathcal{Q}_{\mathrm{RFSI}}$ and $a, b, c, d \in A$. Recall that $\operatorname{Tr}_{\varphi}(x \approx y, z \approx w)$ is just the formula $\forall \bar{u} \varphi(x, y, z, w, \bar{u})$. Thus

$$
\begin{aligned}
\mathbf{A} \vDash \operatorname{Tr}_{\varphi}(x \approx y, z \approx w)[a, b, c, d] & \Leftrightarrow \Theta_{\mathcal{Q}}(a, b) \cap \Theta_{\mathcal{Q}}(c, d)=\Delta_{A} \\
& \Leftrightarrow \Theta_{\mathcal{Q}}(c, d) \cap \Theta_{\mathcal{Q}}(a, b)=\Delta_{A} \\
& \Leftrightarrow \mathbf{A} \vDash \operatorname{Tr}_{\varphi}(z \approx w, x \approx y)[a, b, c, d] .
\end{aligned}
$$

To show $\mathcal{Q}_{\mathrm{RFSI}} \vDash \alpha$ it obviously suffices to show that, for all $\mathbf{A} \in \mathcal{Q}_{\mathrm{RFSI}}$ and all $a, b, c, d, e, f \in \mathbf{A}$,

$$
\begin{aligned}
\mathbf{A} \vDash \operatorname{Tr}_{\varphi}\left(\operatorname{Tr}_{\varphi}(x \approx y, z \approx w),\right. & r \approx s)[a, b, c, d, e, f] \\
& \Leftrightarrow\left(\Theta_{\mathcal{Q}}(a, b) \cap \Theta_{\mathcal{Q}}(c, d)\right) \cap \Theta_{\mathcal{Q}}(e, f)=\Delta_{\mathbf{A}}
\end{aligned}
$$

$$
\begin{aligned}
\mathbf{A} \vDash \operatorname{Tr}_{\varphi}\left(x \approx y, \operatorname{Tr}_{\varphi}(z \approx w,\right. & r \approx s))[a, b, c, d, e, f] \\
& \Leftrightarrow \Theta_{\mathcal{Q}}(a, b) \cap\left(\Theta_{\mathcal{Q}}(c, d) \cap \Theta_{\mathcal{Q}}(e, f)\right)=\Delta_{\mathbf{A}} .
\end{aligned}
$$

To establish these equivalences we have to look at the the internal structure of $\forall \bar{u} \varphi$. Recall that $\operatorname{Tr}_{\varphi}(x \approx y, z \approx w):=\forall \bar{u} \varphi(x, y, z, w, \bar{u})$. If $\forall \bar{u} \varphi$ is of the form (1), then

$$
\operatorname{Tr}_{\varphi}(x \approx y, z \approx w):=\forall \bar{u}\left(\bigwedge_{i<m} \tau_{i}(x, y, z, w, \bar{u}) \approx \sigma_{i}(x, y, z, w, \bar{u})\right),
$$

and thus

$$
\begin{aligned}
\operatorname{Tr}_{\varphi}\left(\operatorname{Tr}_{\varphi}(x \approx y, z \approx w), r \approx s\right) \\
:=\forall \bar{u}\left(\bigwedge_{i<m} \operatorname{Tr}_{\varphi}\left(\tau_{i}(x, y, z, w, \bar{u}) \approx \sigma_{i}(x, y, z, w, \bar{u}), r \approx s\right)\right)
\end{aligned}
$$


Let $\mathbf{A} \in \mathcal{Q}_{\mathrm{RFSI}}$ and $a, b, c, d, e, f \in A$. Then

$$
\mathbf{A} \vDash \operatorname{Tr}_{\varphi}\left(\operatorname{Tr}_{\varphi}(x \approx y, z \approx w), r \approx s\right)[a, b, c, d, e, f]
$$

iff

(9) $\Theta_{\mathcal{Q}}\left(\tau_{i}^{\mathbf{A}}(a, b, c, d, \bar{g}), \sigma_{i}^{\mathbf{A}}(a, b, c, d, \bar{g})\right) \cap \Theta_{\mathcal{Q}}(e, f)=\Delta_{A}$,

for all $i<m$ and $\bar{g} \in A^{n}$.

Since $\mathbf{A}$ is relatively finitely subdirectly irreducible, there are only two cases to consider: (I) $e=f$, i.e., $\Theta_{\mathcal{Q}}(e, f)=\Delta_{\mathbf{A}} ;$ (II) $\tau_{i}^{\mathbf{A}}(a, b, c, d, \bar{g})=\sigma_{i}^{\mathbf{A}}(a, b, c, d, \bar{g})$ for all $i<m$ and $\bar{g} \in A^{n}$, i.e., $\mathbf{A} \vDash \forall \bar{u} \varphi(a, b, c, d, \bar{u})$, which is equivalent to $\Theta_{\mathcal{Q}}(a, b) \cap$ $\Theta_{\mathcal{Q}}(c, d)=\Delta_{\mathbf{A}}$. So (9) is equivalent to $\left(\Theta_{\mathcal{Q}}(a, b) \cap \Theta_{\mathcal{Q}}(c, d)\right) \cap \Theta_{\mathcal{Q}}(e, f)=\Delta_{\mathbf{A}}$. Thus (7) holds, and (8) is established by a similar argument.

LEMMA 5.4. Let $\forall \bar{u} \varphi(x, y, z, w, \bar{u})$ be a conjunction of parameterized equations. Then for every parameterized quasi-equation $\psi(\bar{x})$,

$$
\alpha \vDash L t_{\varphi} \psi \leftrightarrow R t_{\varphi} \psi
$$

PROOF. Let $\psi(\bar{x})$ be $\xi(\bar{x}) \rightarrow \eta(\bar{x})$ where $\xi(\bar{x})$ and $\eta(\bar{x})$ are as in (2) and (3). Then

$$
\begin{aligned}
L t_{\varphi}(\psi(\bar{x})) & :=\operatorname{Tr}_{\varphi}(\psi(\bar{x}), z \approx w) \\
& :=\forall \bar{v}\left(\bigwedge_{i<k} \operatorname{Tr}_{\varphi}\left(\kappa_{i}(\bar{x}, \bar{v}) \approx \lambda_{i}(\bar{x}, \bar{v}), z \approx w\right)\right) \\
& \rightarrow \forall \bar{v}\left(\bigwedge_{j<l} \operatorname{Tr}_{\varphi}\left(\pi_{j}(\bar{x}, \bar{v}) \approx \rho_{j}(\bar{x}, \bar{v}), z \approx w\right)\right) .
\end{aligned}
$$

Under $\alpha$ the last formula is equivalent to

$$
\begin{aligned}
\forall \bar{v}\left(\bigwedge_{i<k} \operatorname{Tr}_{\varphi}\left(z \approx w, \kappa_{i}(\bar{x}, \bar{v}) \approx \lambda_{i}(\bar{x}, \bar{v})\right)\right) & \\
& \rightarrow \forall \bar{v}\left(\bigwedge_{j<l} \operatorname{Tr}_{\varphi}\left(z \approx w, \pi_{j}(\bar{x}, \bar{v}) \approx \rho_{j}(\bar{x}, \bar{v})\right)\right),
\end{aligned}
$$

which by definition is $R t_{\varphi} \psi$.

LEMMA 5.5. Let $\forall \bar{u} \varphi$ be a conjunction of parameterized equations, and let $\psi(\bar{x})$ be a single parameterized quasi-equation. Let $z$ and $w$ be variables not occurring in $\psi(\bar{x})$ (or in $\forall \bar{u} \varphi$ ). Then

$$
\vDash \forall z w\left(\operatorname{Tr}_{\varphi}(\psi(\bar{x}), z \approx w)\right) \rightarrow \operatorname{Tr}_{\varphi}(\psi(\bar{x}), \vartheta(\bar{z}))
$$

for every conjunction $\vartheta(\bar{z})$ of parameterized equations.

PROOF. Let $\psi(\bar{x}):=\xi(\bar{x}) \rightarrow \eta(\bar{x})$ where $\xi(\bar{x})$ and $\eta(\bar{x})$ are conjunctions of parameterized equations, and let $\vartheta(\bar{z}):=\forall \bar{v}\left(\bigwedge_{i<k} \kappa_{i}(\bar{z}, \bar{v}) \approx \lambda_{i}(\bar{z}, \bar{v})\right)$. Then $\operatorname{Tr}_{\varphi}(\psi(\bar{x}), \vartheta(\bar{z}))$ is logically equivalent to

$$
\forall \bar{v}\left(\bigwedge_{i<k} \operatorname{Tr}_{\varphi}\left(\xi(\bar{x}), \kappa_{i}(\bar{z}, \bar{v}) \approx \lambda_{i}(\bar{z}, \bar{v})\right)\right) \rightarrow \forall \bar{v}\left(\bigwedge_{i<k} \operatorname{Tr}_{\varphi}\left(\eta(\bar{x}), \kappa_{i}(\bar{z}, \bar{v}) \approx \lambda_{i}(\bar{z}, \bar{v})\right)\right) .
$$


However this is logically implied by

$$
\forall \bar{v}\left[\bigwedge_{i<k} \operatorname{Tr}_{\varphi}\left(\xi(\bar{x}), \kappa_{i}(\bar{z}, \bar{v}) \approx \lambda_{i}(\bar{z}, \bar{v})\right) \rightarrow \operatorname{Tr}_{\varphi}\left(\eta(\bar{x}), \kappa_{i}(\bar{z}, \bar{v}) \approx \lambda_{i}(\bar{z}, \bar{v})\right)\right],
$$

which in fact coincides with

$$
\forall \bar{v}\left[\bigwedge_{i<k} \operatorname{Tr}_{\varphi}\left(\psi(\bar{x}), \kappa_{i}(\bar{z}, \bar{v}) \approx \lambda_{i}(\bar{z}, \bar{v})\right)\right] .
$$

The expression within the conjunction symbol is a substitution instance of $\forall z w\left(\operatorname{Tr}_{\varphi}(\psi(\bar{x}), z \approx w)\right)$.

LEMMA 5.6. Let $\forall \bar{u} \varphi(x, y, z, w, \bar{u})$ be a conjunction of parameterized equations. Then for every parameterized quasi-equation $\psi, \beta$,

$$
L t_{\varphi} \psi \vDash L t_{\varphi} L t_{\varphi} \psi
$$

Proof. Let $\psi(\bar{x})$ be $\xi(\bar{x}) \rightarrow \eta(\bar{x})$ where $\xi(\bar{x})$ and $\eta(\bar{x})$ are as in (2) and (3). $L t_{\varphi} L t_{\varphi} \psi$ is logically equivalent to

$$
\begin{aligned}
& \forall \bar{v}\left[\bigwedge _ { i < k } \operatorname { T r } _ { \varphi } \left(\operatorname { T r } _ { \varphi } \left(\kappa_{i}(\bar{x}, \bar{v})\right.\right.\right.\left.\left.\left.\approx \lambda_{i}(\bar{x}, \bar{v}), z \approx w\right), r \approx s\right)\right] \\
& \rightarrow \forall \bar{v}\left[\bigwedge_{j<l} \operatorname{Tr}_{\varphi}\left(\operatorname{Tr}_{\varphi}\left(\pi_{j}(\bar{x}, \bar{v}) \approx \rho_{j}(\bar{x}, \bar{v}), z \approx w\right), r \approx s\right)\right] .
\end{aligned}
$$

Under the assumption $\beta$ the conjunct on the left is equivalent to

$$
\operatorname{Tr}_{\varphi}\left(\kappa_{i}(\bar{x}, \bar{v}) \approx \lambda_{i}(\bar{x}, \bar{v}), \operatorname{Tr}_{\varphi}(z \approx w, r \approx s)\right),
$$

and the conjunct on the right is equivalent to

$$
\operatorname{Tr}_{\varphi}\left(\pi_{j}(\bar{x}, \bar{v}) \approx \rho_{j}(\bar{x}, \bar{v}), \operatorname{Tr}_{\varphi}(z \approx w, r \approx s)\right) .
$$

Thus $L t_{\varphi} L t_{\varphi} \psi$ is equivalent under $\beta$ to $\operatorname{Tr}_{\varphi}\left(\psi(\bar{x}), \operatorname{Tr}_{\varphi}(z \approx w, r \approx s)\right)$. But by Lemma 5.5 this last formula is a consequence of $L t_{\varphi} \psi$ (taking $\operatorname{Tr}_{\varphi}(z \approx w, r \approx s$ ) for $\vartheta(\bar{x}))$.

\section{The main results.}

THEOREM 6.1. Let $\mathcal{Q}$ be a finitely generated and relatively congruence-distributive quasivariety. There exists a finite set $Q$ of quasi-identities such that $\mathcal{Q}=$ $\operatorname{Mod}(Q \cup E)$, where $E$ is any base for the identities of $\mathcal{Q}$.

By hypothesis $\mathcal{Q}$ is finitely generated, and hence by the relativized Jónsson lemma $\mathcal{Q}_{\mathrm{RFSI}}$ is up to isomorphism a finite set of finite algebras. So it is elementary. This together with relative congruence-distributivity implies that $\mathcal{Q}$ has parameterized REDPM (Theorem 2.2). So there exists a conjunction $\forall \bar{u} \varphi(x, y, z, w, \bar{u})$ of parameterized quasi-equations that defines the intersection of principal $\mathcal{Q}$-congruence meets. Let $\mathcal{Q}:=\operatorname{Mod} \Gamma$, with $\Gamma$ a possibly infinite set of quasi-identities. Then

$$
\mathcal{Q}_{\mathrm{RFSI}}=\operatorname{Mod}(\Gamma \cup\{\forall x y z w(\forall \bar{u} \varphi(x, y, z, w, \bar{u}) \rightarrow x \approx y \wedge z \approx w)\}),
$$

and, since $\mathcal{Q}_{\mathrm{RFSI}}$ is finitely axiomatizable, there exists a finite $\Gamma^{\prime} \subseteq \Gamma$ such that

$$
\mathcal{Q}_{\mathrm{RFSI}}=\operatorname{Mod}\left(\Gamma^{\prime} \cup\{\forall x y z w(\forall \bar{u} \varphi(x, y, z, w, \bar{u}) \rightarrow x \approx y \wedge z \approx w)\}\right) .
$$


Let $\Sigma$ be the set of quasi-equations characterizing congruence relations, viz., $x \approx x, x \approx y \rightarrow y \approx x, x \approx y \wedge y \approx z \rightarrow x \approx z$, and $\left(\bigwedge_{i<m} x_{i} \approx y_{i}\right) \rightarrow$ $\omega x_{0}, \ldots, x_{m-1} \approx \omega y_{0}, \ldots, y_{m-1}$ for every operation symbol $\omega . \Sigma$ is finite because the language type is assumed finite. We also define

$$
\begin{gathered}
\gamma:=\forall \bar{u} \varphi(x, y, x, y, \bar{u}) \rightarrow x \approx y, \\
\Omega:=\{\alpha, \beta, \gamma\} \cup L t_{\varphi}\left(\Gamma^{\prime} \cup\{\alpha, \beta, \gamma\} \cup \Sigma\right),
\end{gathered}
$$

where $\alpha$ and $\beta$ are the parameterized quasi-equations defined in (5) and (6) of $\S 5$. For any set $\Delta$ of parameterized quasi-equations, let $L t_{\varphi} \Delta=\left\{L t_{\varphi} \psi: \psi \in \Delta\right\}$, and similarly for $R t_{\varphi} \Delta$. We also write $\Delta \vDash \Lambda$ as shorthand for $\Delta \vDash \psi$ for every $\psi \in \Lambda$, where $\Lambda$ is any set of parameterized quasi-equations.

LEMMA 6.2. $\Omega \vDash L t_{\varphi}\left(\Gamma^{\prime} \cup \Omega\right) \cup R t_{\varphi}\left(\Gamma^{\prime} \cup \Omega\right)$.

ProOF. Let $\psi \in \Gamma^{\prime} \cup \Omega:=\Gamma^{\prime} \cup\{\alpha, \beta, \gamma\} \cup L t_{\varphi}\left(\Gamma^{\prime} \cup\{\alpha, \beta, \gamma\} \cup \Sigma\right)$. If $\psi \in \Gamma^{\prime} \cup\{\alpha, \beta, \gamma\}$, then $L t_{\varphi} \psi \in \Omega$ by definition of $\Omega$. Thus $\Omega \vDash L t_{\varphi} \psi$ trivially, and hence $\Omega \vDash R t_{\varphi} \psi$ since $\alpha, L t_{\varphi} \psi \vDash R t_{\varphi} \psi$ by Lemma 5.4. Suppose $\psi=L t_{\varphi} \vartheta$ with $\vartheta \in \Gamma^{\prime} \cup\{\alpha, \beta, \gamma\} \cup \Sigma$. Then $\beta, \psi \vDash L t_{\varphi} \psi$ by Lemma 5.6, and thus also $\alpha, \beta, \psi \vDash R t_{\varphi} \psi$ since $\alpha, L t_{\varphi} \psi \vDash R t_{\varphi} \psi$. Therefore $\Omega \vDash L t_{\varphi} \psi$ and $\Omega \vDash R t_{\varphi} \psi$.

Let

$$
\mathcal{L}:=\operatorname{Mod}\left(\Gamma^{\prime} \cup \Omega\right) \text {. }
$$

$\mathcal{L}$ is a parameterized quasivariety, and thus the notions of $\mathcal{L}$-congruence and finite subdirect irreducibility relative to $\mathcal{L}$ are well defined; see $\S 4$.

LEMMA 6.3. $\mathcal{L}_{\mathrm{RFSI}} \subseteq \mathcal{Q}_{\mathrm{RFSI}}$. show

ProOf. Let $\mathbf{A} \in \mathcal{L}_{\mathrm{RFSI}} . \mathbf{A} \vDash \Gamma^{\prime}$, so in order to show $\mathbf{A} \in \mathcal{Q}_{\mathrm{RFSI}}$ it suffices to

$$
\mathbf{A} \vDash \forall x y z w(\forall \bar{u} \varphi(x, y, z, w, \bar{u}) \rightarrow x \approx y \wedge z \approx w) .
$$

Since $\mathbf{A} \in \mathcal{L}_{\mathrm{RFSI}}$, we have that $\Phi \cap \Psi=\Delta_{A}$ implies $\Phi=\Delta_{A}$ or $\Psi=\Delta_{A}$, for all $\Phi, \Psi \in \operatorname{Con}_{\mathcal{L}} \mathbf{A}$.

Let $a, b, c, d \in A$ such that $\mathbf{A} \vDash \forall \bar{u} \varphi(a, b, c, d, \bar{u})$. Define

$$
\Phi:=\{\langle e, f\rangle: \mathbf{A} \vDash \forall \bar{u} \varphi(e, f, c, d, \bar{u})\} .
$$

Let $\psi$ be any parameterized quasi-equation in $\Gamma^{\prime} \cup \Omega$. Since $\Omega \vDash L t_{\varphi} \psi$, we have by Lemma 5.1 that $\Phi$ is closed under $\psi$. Thus $\Phi$ is a $\mathcal{L}$-congruence; observe that by assumption $\langle a, b\rangle \in \Phi$. Now define

$$
\boldsymbol{\Psi}:=\{\langle g, h\rangle: \mathbf{A} \vDash \forall \bar{u} \varphi(e, f, g, h, \bar{u}) \text { for all }\langle e, f\rangle \in \Phi\} .
$$

The fact that $\Omega \vDash R t_{\varphi} \psi$ for every $\psi \in \Gamma^{\prime} \cup \Omega$ implies $\Psi$ is a $\mathcal{L}$-congruence, and, by definition of $\Phi,\langle c, d\rangle \in \Psi$. Let $\langle e, f\rangle \in \Phi \cap \Psi$. Then $\mathbf{A} \vDash \forall \bar{u} \varphi(e, f, e, f, \bar{u})$. Thus $e=f$ since $\mathbf{A} \vDash \gamma$. So $\Phi \cap \Psi=\Delta_{A}$. This implies either $\Phi=\Delta_{A}$ or $\Psi=\Delta_{A}$, and hence either $a=b$ or $c=d$. So (1) is established.

We are ready now to prove the theorem. We first show that $\mathcal{Q} \subseteq \mathcal{L}$. Recall that $\mathcal{L}=\operatorname{Mod}\left(\Gamma^{\prime} \cup \Omega\right)$, where $\Omega=\{\alpha, \beta, \gamma\} \cup L t_{\varphi}\left(\Gamma^{\prime} \cup\{\alpha, \beta, \gamma\} \cup \Sigma\right)$. Trivially $\mathcal{Q} \vDash \Gamma^{\prime}$ since $\Gamma^{\prime} \subseteq \Gamma$ and $\Gamma$ is an axiom set for $\mathcal{Q}$. Because $\forall \bar{u} \varphi$ defines meets of principal $\mathcal{Q}$-congruences in $\mathcal{Q}$, we get $\mathcal{Q} \vDash \alpha, \beta, \gamma$. (This was shown for $\alpha$ and $\beta$ in Lemma 5.3; 
for $\gamma$ see the proof of 3.4). For the same reason we have $\mathcal{Q} \vDash L t_{\varphi}\left(\Gamma^{\prime} \cup\{\alpha, \beta, \gamma\} \cup \Sigma\right)$ by Lemma 5.2 ( $\mathcal{Q} \vDash \Sigma$ since the quasi-identities in $\Sigma$ are all logically true).

Thus $\Gamma \vDash \Gamma^{\prime} \cup \Omega$. Since $\Gamma^{\prime} \cup \Omega$ is finite, there exists a finite subset $Q$ of $\Gamma$ such that $Q \vDash \Gamma^{\prime} \cup \Omega$. Then $\mathcal{Q} \subseteq \operatorname{Mod}(Q \cup E) \subseteq \mathcal{L}$, where $E$ is any base for the identities of $\mathcal{Q}$. To complete the proof we show that $\operatorname{Mod}(Q \cup E) \subseteq \mathcal{Q}$.

$\operatorname{Mod}(Q \cup E)$ is a subquasivariety of the variety $\operatorname{Mod} E$ generated by $\mathcal{Q}$, which is finitely generated by hypothesis. Thus $\operatorname{Mod}(Q \cup E)$ is locally finite. So it suffices to prove that every finite member of $\operatorname{Mod}(Q \cup E)$ is contained in $\mathcal{Q}$.

Let $\mathbf{A} \in \operatorname{Mod}(Q \cup E)$, where $\mathbf{A}$ is finite. Since $\mathbf{A} \in \mathcal{L}$ and $\mathbf{A}$ is finite, $\mathbf{A} \cong$ $\mathbf{B} \subseteq \mathrm{SD} \mathbf{C}_{0} \times \mathbf{C}_{1} \times \cdots \times \mathbf{C}_{n-1}$ with $\mathbf{C}_{i} \in \mathcal{L}_{\mathrm{RFSI}}$ for each $i<n$. By Lemma 6.3 $\mathbf{C}_{i} \in \mathcal{Q}_{\mathrm{RFSI}}$ for each $i$. Thus $\mathbf{A} \in \mathcal{Q}$. This completes the proof of Theorem 6.1.

We are now finally ready to prove Theorem I, the quasivariety analogue of Baker's theorem.

THEOREM I. Every finitely generated and relatively congruence-distributive quasivariety is finitely based.

PROOF. Let $\mathcal{Q}$ be a quasivariety satisfying the hypothesis of the theorem. By Theorem 6.1, $\mathcal{Q}=\operatorname{Mod}(Q \cup E)$, where $Q$ is a finite set of quasi-identities, and $E$ is a possibly infinite set of identities. Let $\mathcal{Q}^{\prime}:=\operatorname{Mod} Q$. Then $\mathcal{Q}^{\prime}$ is a finitely based quasivariety, and $\mathcal{Q}$ is a relative subvariety of $\mathcal{Q}^{\prime}$. Thus the hypothesis of Theorem 3.1 is satisfied, and, since $\mathcal{Q}$ is relatively congruence-distributive, and finitely generated (so that $\mathcal{Q}_{\text {RFSI }}$ is strictly elementary), we may apply 3.1 to conclude that $\mathcal{Q}$ is finitely based.

As an almost immediate corollary of this theorem we get Theorem II, the analogue of Baker's theorem for relative subvarieties. It improves Corollary 3.3 by removing the premiss that the quasivariety $\mathcal{Q}$ be finitely based.

THEOREM II. Let $\mathcal{Q}$ be a relatively congruence-distributive quasivariety. Then every finitely generated relative subvariety of $\mathcal{Q}$ is finitely based.

PROOF. Let $R$ be a finitely generated subvariety of $\mathcal{Q}$. Let $\mathcal{M}$ be any finite set of algebras generating $R$. By the second version of the relativized Jónsson lemma (Theorem 1.7), $R_{\mathrm{RFSI}} \subseteq \mathrm{H}_{\mathcal{Q}} \mathrm{SP}_{\mathrm{U}} \mathcal{M} \subseteq \mathrm{H}_{\mathcal{Q}} \mathrm{S} \mathcal{M}$. So $R$ is also finitely generated as quasivariety. It is also relatively congruence-distributive since it is a relative subvariety of a quasivariety with the property. Thus $R$ is finitely based by Theorem I.

7. Relatively congruence-distributive quasivarieties. The applicability of the various finite basis results discussed in the preceding sections is limited, at least in comparison with analogous results for varieties, by the difficulty encountered in establishing relative congruence-distributivity in concrete situations. No condition like the existence of Jónsson terms is known to characterize the property. And such a condition, if it does exist, would likely be radically different from the familiar $\mathrm{Mal}^{\prime} \mathrm{cev}$-style conditions since relative congruence-distributivity is not inherited by subquasivarieties. However several general methods of establishing relative congruence-distributivity are now available that taken together prove to be quite effective in many cases.

W. Dziobiak has established the following two important results. 
THEOREM 7.1 [15]. If $\mathcal{Q}$ is a relatively congruence-distributive quasivariety, then $\mathcal{Q}_{\mathrm{RFSI}}=\mathcal{Q}_{\mathrm{FSI}}$, i.e., every relatively finitely subdirectly irreducible member of $\mathcal{Q}$ is finitely subdirectly irreducible in the absolute sense.

COROLLARY $7.2[\mathbf{1 5}]$. If $\mathcal{Q}$ is a subquasivariety of a relatively congruence-distributive quasivariety, then $\mathcal{Q}$ itself is relatively congruence-distributive iff $\mathcal{Q}_{\mathrm{RFSI}}$ $=\mathcal{Q}_{\mathrm{FSI}}$.

In particular, any quasivariety $\mathcal{Q}$ that is known to generate a congruence-distributive variety, a quasivariety of lattices for instance, is relatively congruence-distributive just in case all its relatively finitely subdirectly irreducible members are absolutely finitely subdirectly irreducible. If $\mathcal{Q}$ is finitely generated there is an effective procedure for deciding if this latter condition holds: List all subalgebras of members of the generating set, and check each one if it is isomorphic to a proper subdirect product of other algebras in the list; by the relative Jónsson lemma (1.7) the ones that fail this test are the relatively finitely subdirectly irreducibles. Then check each of the latter to see if it is absolutely subdirectly irreducible. When $\mathcal{Q}$ is generated by just a few small finite algebras this can also be a practical procedure. For example Dziobiak uses it in [15] to show that a certain 12-element nonmodular lattice generates a relatively congruence-distributive quasivariety that is distinct from the variety it generates. This settles negatively a question raised by Tumanov [35] whether a finite lattice generates a finitely based quasivariety just in case the generated quasivariety coincides with the generated variety. ${ }^{2}$

For a large class of congruence-distributive varieties there is an even more effective method, due to Czelakowski and Dziobiak [12], for establishing the relative congruence distributivity of subquasivarieties.

A quasivariety is said to have equationally definable relative principal congruence meets (REDPM) if it has parameterized REDPM with an empty list of parameters, i.e., if the equivalent conditions 2.1(1),(2),(3) hold for some conjunction of equations without parameters

$$
\varphi(x, y, z, w):=\bigwedge_{i<m} \tau_{i}(x, y, z, w) \approx \sigma_{i}(x, y, z, w) .
$$

This property, applied exclusively to varieties, was considered first by Baker [1] and later by Blok and Pigozzi [8] where it is called EDPM. There are a large number of varieties with the property (see §9). Czelakowski and Dziobiak [12] were the first to relativize the property to quasivarieties and investigate its consequences. They have obtained the following counterpart of Theorem 2.2; recall that a class of algebras is universal if it is elementary and closed under the formation of subalgebras (or, equivalently, if it is definable by an arbitrary set of universal first-order sentences).

THEOREM 7.3 [12]. For any quasivariety $\mathcal{Q}$ the following are equivalent:

(1) $\mathcal{Q}$ has REDPM;

(2) $\mathcal{Q}$ is relatively congruence-distributive, and $\mathcal{Q}_{\mathrm{RFSI}}$ is a universal class;

(3) $\mathcal{Q}$ is relatively congruence-distributive, and the compact (i.e., finitely generated) $\mathcal{Q}$-congruences are closed under intersection.

The special case of this theorem for varieties was first proved in [8].

\footnotetext{
${ }^{2}$ Tumanov raised the same question for modular lattices. This is still open.
} 
THEOREM 7.4 [12]. If $\mathcal{Q}$ is a subquasivariety of a variety with EDPM, then the following are equivalent.

(1) $\mathcal{Q}$ has REDPM;

(2) $\mathcal{Q}$ is relatively congruence-distributive;

(3) $\mathcal{Q}$ is generated by a class of absolutely finitely subdirectly irreducible algebras.

So any set of finitely subdirectly irreducible algebras from a variety with EDPM generates a relatively congruence-distributive quasivariety. This is not the case for arbitrary congruence-distributive varieties. [12] contains an example of a finite, absolutely subdirectly irreducible lattice whose generated subquasivariety fails to be relatively congruence-distributive. In fact it is not finitely based.

The two methods of establishing relative congruence-distributivity described above can be applied only to quasivarieties that are known beforehand to generate congruence-distributive varieties. There are other methods however that do not have this limitation. One can try to show $\mathcal{Q}$ has REDPM by finding a conjunction $\varphi(x, y, z, w)$ of equations and verifying directly that

$$
\mathcal{Q}_{\mathrm{RFSI}} \vDash \forall x y z w(\varphi(x, y, z, w) \leftrightarrow x \approx y \vee z \approx w) ;
$$

more generally one can try to show $\mathcal{Q}$ has parameterized REDPM by verifying (1) for some conjunction of parameterized equations. (See Theorem 2.2.) Again this can be a practical course to take if $\mathcal{Q}$ is finitely generated so that the relatively finitely subdirectly irreducibles can be identified in the manner described previously.

Another effective method of establishing relative congruence distributivity is to show $\mathcal{Q}$ is a relative subvariety of a quasivariety that is known to have the property. This is the method we shall use in the following subsection to construct a large class of finite algebras that individually generate finitely based quasivarieties and nonfinitely based varieties.

Equality-test algebras. Let $\mathbf{A}=\left\langle A, \omega^{\mathbf{A}}\right\rangle_{\omega \in T}$ be an algebra of arbitrary type $T$ with at least two distinct elements. Form a new algebra Et $\mathbf{A}$ by adjoining new operations $0,1,+, \cdot,-$, and $e q$ to $\mathbf{A}$ that are defined as follows: 0 and 1 are distinct fixed but arbitrary elements of $A$. For all $a, b \in A$,

$$
\begin{array}{rlrl}
a+b & := \begin{cases}0 & \text { if } a=b=0, \\
1 & \text { otherwise }\end{cases} & a \cdot b: & : \begin{cases}0 & \text { if } a=0 \text { or } b=0 \\
1 & \text { otherwise }\end{cases} \\
-a & := \begin{cases}1 & \text { if } a=0, \\
0 & \text { otherwise; }\end{cases} & e q(a, b):= \begin{cases}1 & \text { if } a=b, \\
0 & \text { otherwise }\end{cases}
\end{array}
$$

Observe that $\mathbf{A}$ coincides with the $T$-reduct of $\mathbf{E t} \mathbf{A}$, and that the two-element Boolean algebra is a subalgebra of the $\{+, \cdot,-, 0,1\}$-reduct of Et A. Moreover, this two-element Boolean subreduct is definable in $\mathbf{E t} \mathbf{A}$ as the range of the operation - (and also as the range of each of,$+ \cdot$, and $e q$ ). This provides a way of defining in a canonical way a Boolean subreduct of every algebra in the variety generated by $\mathbf{E t} \mathbf{A}$.

Et $\mathbf{A}$ is called the (Boolean) equality-test algebra on $\mathbf{A}$; it is uniquely determined up to the choice of 0 and 1 . Et $\mathbf{A}$ can be viewed as the homogeneous counterpart of a heterogeneous algebra with two sorts: one corresponding to the original algebra $\mathbf{A}$, and the other to the two-element Boolean algebra. The equality test $e q$ is the only inter-sort operation. Heterogeneous algebras of this kind, with possibly $\mathbf{A}$ 
itself taken to be a heterogeneous algebra, arise naturally in the theory of abstract data structures. They will be discussed in the next section.

We will prove that the quasivariety generated by $\mathbf{E t} \mathbf{A}$ is relatively congruencedistributive. We do this by characterizing axiomatically the quasivariety generated by all equality-test algebras and showing it is relatively congruence-distributive. We then show $Q v(\mathbf{E t} \mathbf{A})$ is a relative subquasivariety.

The quasivariety $\mathcal{G E} T$ of generalized equality-test algebras is defined by four groups of identities and quasi-identities; in these axioms $\tau \leq \sigma$ is an abbreviation for $\tau+\sigma \approx \sigma$ :

$$
\begin{gathered}
x+y \approx-(-x \cdot-y), \quad x \cdot y \approx-(-x+-y), \\
0 \approx-1, \quad 1 \approx-0, \\
e q(x, y) \approx--e q(x, y) ; \\
x+y \approx y+x, \quad x \cdot y \approx y \cdot x, \\
x+(y \cdot z) \approx(x+y) \cdot(x+z), \quad x \cdot(y+z) \approx(x \cdot y)+(x \cdot z), \\
-x+0 \approx-x, \quad-x \cdot 1 \approx-x, \\
x+-x \approx 1, \quad x \cdot-x \approx 0 ; \\
e q(x, x) \approx 1, \\
e q(x, y) \approx e q(y, x), \\
e q(x, y) \cdot e q(y, z) \leq e q(y, z), \\
e q\left(x_{0}, y_{0}\right) \cdot \ldots \cdot e q\left(x_{m-1}, y_{m-1}\right) \leq e q\left(\omega x_{0} \ldots x_{m-1}, \omega y_{0} \ldots y_{m-1}\right) \\
\quad \text { for every } \omega \in T \cup\{+, \cdot,-e q\} ;
\end{gathered}
$$

$$
\begin{gathered}
e q(x, 0) \approx-x . \\
e q(-x, 1) \approx-x . \\
e q(x, y) \approx 1 \rightarrow x \approx y .
\end{gathered}
$$

The axioms of group (I) assure that the range of - includes 0 and 1 and the ranges of the operations $+, \cdot,-$, and $e q$. Group (II) axioms guarantee that the range of - forms a Boolean algebra. The axioms of groups (III) and (IV) connect $\mathcal{G E} \tau$ congruences with Boolean filters, as we see below. Observe that all the axioms are identities except the last one. Thus in checking that a congruence is a $\mathcal{G E} T$ congruence one only needs to verify closure under this single quasi-identity.

It is an easy matter to check that $\mathbf{E t} \mathbf{A} \in \mathcal{G E} \tau$ for every algebra $\mathbf{A}$ (of the appropriate type). Consider for example the last axiom of (III). Let $a_{0}, \ldots, b_{m-1} \in$ $A$. Then $e q\left(a_{0}, b_{0}\right) \cdot \ldots \cdot e q\left(a_{m-1}, b_{m-1}\right)$ equals 1 if $a_{i}=b_{i}$ for all $i<m$; otherwise it equals 0 . In both cases it is no larger than $e q\left(\omega^{\mathbf{A}}(\bar{a}), \omega^{\mathbf{A}}(\bar{b})\right)$. To see that the first identity of (IV) holds observe that, if $a \neq 0$, then $e q(a, 0)=0=-a$. Otherwise $e q(a, 0)=1=-a$.

Consider any algebra $\mathbf{E} \in \mathcal{G E} T$, and let $V:=\{-a: a \in E\}$, i.e., the range of -. The algebra $\mathbf{V}:=\langle V,+, \cdot,-, 0,1\rangle$ is called the value algebra of $\mathbf{E}$. As previously noted $\mathbf{V}$ is a Boolean algebra. For any $\Phi \in \operatorname{Con}_{\mathcal{E}} \tau \mathbf{E}$, define

$$
F i \Phi:=\{a \in V: a \equiv 1(\bmod \Phi)\} \text {. }
$$


For any $a, b \in F i \Phi, a \cdot b \equiv 1 \cdot 1=1(\bmod \Phi)$, and for all $a \in F i \Phi$ and $b \in E$ such that $a \leq b, b=a+b \equiv 1+b=1(\bmod \Phi)$. Thus $F i \Phi$ is a filter of $\mathbf{V}$.

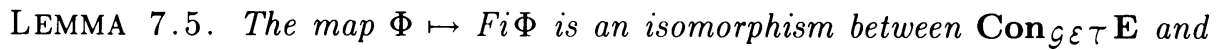
the lattice of filters of $\mathrm{V}$.

PROOF. Let $\Phi$ be any $\mathcal{G} \mathcal{E} T$-congruence, and consider any $a, b \in E$. We show that $a \equiv b(\bmod \Phi)$ iff $e q(a, b) \in F i \Phi$. If $a \equiv b(\bmod \Phi)$, then $e q(a, b) \equiv e q(a, a)=1$ $(\bmod \Phi)$; if $e q(a, b) \equiv 1(\bmod \Phi)$, then $a \equiv b(\bmod \Phi)$ since $\Phi$, as a $\mathcal{G} \mathcal{E} \tau$-congruence, is closed under $e q(x, y) \approx 1 \rightarrow x \approx y$. Thus the map $\Phi \mapsto F i \Phi$ is one-one.

Let $G$ be any filter of $\mathbf{V}$, and define

$$
\Phi:=\{\langle a, b\rangle \in E \times E: e q(a, b) \in G\} .
$$

We prove that $\Phi$ is a $\mathcal{G} \mathcal{E} T$-congruence such that $F i \Phi=G$. This will show that $F i$ is a bijection between $C_{\mathcal{G}} \tau \mathbf{E}$ and the filter lattice of $\mathbf{V}$, and thus complete the proof of the lemma since $F i$ is clearly order-preserving.

The axioms of group (III) guarantee that $\Phi$ is a congruence of $\mathbf{E}$. To see it is a $\mathcal{G E} T$-congruence we have to show it is closed under the quasi-identity $e q(x, y) \approx$ $1 \rightarrow x \approx y$. Suppose $e q(a, b) \equiv 1(\bmod \Phi)$. Then $e q(e q(a, b), 1) \in G$ by definition of $\Phi$. But $e q(e q(a, b), 1)=e q(a, b)$ by the third identity of (I) and the second identity of (IV). Thus $e q(a, b) \in G$, and hence $a \equiv b(\bmod \Phi)$. So $\Phi$ is a $\mathcal{G} \mathcal{E} T$ congruence. To see that $F i \Phi=G$ consider any $a \in V$. Then, since $e q(a, 1)=a$, we have $a \in G$ iff $e q(a, 1) \in G$ iff $a \equiv 1(\bmod \Phi)$ iff $a \in F i \Phi$.

It follows immediately from the lemma that $\mathcal{G E} T$ is relatively congruencedistributive.

A generalized equality-test algebra $\mathbf{E}$ is relatively simple if $\mathbf{E}$ is nontrivial and has just two $\mathcal{G} \mathcal{E} T$ - congruences, $\Delta_{E}$ and $\nabla_{E}$. By the next theorem $\mathcal{G} \mathcal{E} T$ is relatively semisimple in the obvious sense.

THEOREM 7.6. Iet $\mathbf{E} \in \mathcal{G E} T$. The following are equivalent.

(1) $\mathbf{E}$ is relatively subdirectly irreducible;

(2) $\mathbf{E}$ is relatively finitely subdirectly irreducible;

(3) $\mathbf{E}$ is relatively simple;

(4) the value algebra of $\mathbf{E}$ is the two-element Boolean algebra;

(5) $\mathbf{E}$ is an equality-test algebra.

If $\mathbf{E}$ is nontrivial, then each of the above conditions is equivalent to

(6) $\mathbf{E} \vDash \forall x y z w(e q(x, y)+e q(z, w) \approx 1 \rightarrow x \approx y \vee z \approx w)$.

ProOF. The equivalence of parts (1)--(4) follows immediately from the lemma and well-known properties of Boolean algebras.

We show parts (4) and (6) are equivalent. Let $\mathbf{V}$ be the value algebra of $\mathbf{E}$. If $V=\{0,1\}$, then (6) holds by the axiom $e q(x, y) \approx 1 \rightarrow x \approx y$. Conversely, assume (6) holds and that $\mathbf{E}$ is nontrivial. Let $b \in V$, so that $b=-a$ for some $a \in E$. By the axioms $e q(x, 0) \approx-x$ and $e q(-x, 1) \approx-x$ we get $e q(b, 0)+e q(b, 1)=$ $e q(-a, 0)+e q(-a, 1)=--a+-a=-b+b=1$. Thus $b=0$ or $b=1$, and $V=\{0,1\}$.

We complete the proof by showing the equivalence of parts (4) and (5). If $\mathbf{E}$ is an equality-test algebra, then $V=\{0,1\}$ by definition, so (5) implies (4). For the reverse implication assume $V=\{0,1\}$. We show that $\mathbf{E}=\mathbf{E t} \mathbf{A}$ where $\mathbf{A}$ is is the 
$T$-reduct of $\mathbf{E}$, i.e, that the equality-test and Boolean operations of $\mathbf{E}$ and $\mathbf{E t} \mathbf{A}$ coincide. Let $a, b \in E$. $e q^{\mathbf{E}}(a, b) \in\{0,1\}$. Thus the two axioms $e q(x, x) \approx 1$ and $e q(x, y) \approx 1 \rightarrow x \approx y$ combine to give

$$
e q(a, b):= \begin{cases}1 & \text { if } a=b \\ 0 & \text { otherwise. }\end{cases}
$$

Using this description of $e q^{\mathbf{E}}$ and the axiom $e q(x, 0) \approx-x$ we get

$$
-a:= \begin{cases}1 & \text { if } a=0, \\ 0 & \text { otherwise. }\end{cases}
$$

The same axiom combined with $x+y \approx-(-x \cdot-y)$ gives $a+b=-(-a \cdot-b)=$ $-(e q(a, 0) \cdot e q(b, 0))$. Thus

$$
a+b:= \begin{cases}0 & \text { if } a=b=0 \\ 1 & \text { otherwise. }\end{cases}
$$

Finally, $e q(x, 0) \approx-x$ and $x \cdot y \approx-(-x+-y)$ combine in a similar manner to give

$$
a \cdot b:= \begin{cases}0 & \text { if } a=0 \text { or } b=0, \\ 1 & \text { otherwise } \quad \square\end{cases}
$$

COROllary 7.7. $\mathcal{G} \mathcal{E} T=\operatorname{ISP}\{\mathbf{E t} \mathbf{A}: \mathbf{A}$ an algebra of type $T\}$.

PROOF. Apply the relativized version of Birkoff's subdirect representation theorem (Theorem 1.1).

Thus $\mathcal{G E} T$ is the quasivariety generated by all equality-test algebras.

COROLLARY 7.8. Let $\mathcal{K}$ be any class of equality-test algebras. Then

$$
V a_{\mathcal{G}} \tau \mathcal{K}=Q v K=\operatorname{ISPP}_{\mathrm{U}} \mathrm{K} \text {. }
$$

PROOF. By the second version of the Jónsson lemma (Theorem 1.7),

$$
\left(V a_{\mathcal{G} \mathcal{E} \tau} \mathcal{K}\right)_{\mathrm{RFSI}} \subseteq \mathrm{H}_{\mathcal{G E} \tau} \mathrm{SP}_{\mathrm{U}} \mathcal{K} .
$$

Since the equality-test algebras form a universal class, each member of $\mathrm{SP}_{U} \mathcal{K}$ is again an equality-test algebra and hence relatively simple. Thus $\left(\operatorname{Vag}_{\mathcal{E}} \tau \mathcal{K}\right)_{\mathrm{RFSI}} \subseteq$ $\mathrm{ISP}_{\mathrm{U}} \mathrm{K} \subseteq Q v \mathrm{~K}$.

THEOREM 7.9. Let $\mathbf{A}$ be an arbitrary algebra.

(1) Et $\mathbf{A}$ generates a relatively congruence-distributive quasivariety.

(2) If the principal filter $\left[\Theta^{\mathbf{A}}(0,1)\right)$ generated by $\Theta^{\mathbf{A}}(0,1)$ in the congruence lattice $\mathbf{C o n} \mathbf{A}$ is not distributive, then $\mathbf{E t} \mathbf{A}$ does not generate a congruence-distributive variety.

PROOF. (1) $Q v(\mathbf{E t} \mathbf{A})$ is a relative subvariety of a relatively congruence-distributive quasivariety and hence is itself relatively congruence-distributive.

(2) It is easy to see that, if $\mathbf{A}$ is nontrivial, $\operatorname{Con}(\mathbf{E t} \mathbf{A})$ is isomorphic to $1 \oplus$ $\left[\Theta^{\mathbf{E}}(0,1)\right)$ : Let $\Phi$ be any congruence on Et $\mathbf{A}$ distinct from $\Delta_{A}$, so that $a \equiv b(\bmod$ $\Phi)$ for some pair $a, b$ of distinct elements of $A$. Then $0=e q(a, b) \equiv e q(a, a)=1$ $(\bmod \Phi)$. So $\Theta^{\mathbf{E}}(0,1) \subseteq \Theta^{\mathbf{E t} \mathbf{A}}(0,1) \subseteq \Phi$. On the other hand, let $\Phi$ be any congruence on $\mathbf{A}$ such that $0 \equiv 1(\bmod \Phi)$. Then, since $a+b, a \cdot b,-a, e q(a, b) \in$ $\{0,1\}$ for all $a, b \in A, \Phi$ is also a congruence of Et $\mathbf{A}$. Thus under the hypothesis of part (2), the variety generated by $\mathbf{E t} \mathbf{A}$ is not congruence-distributive.

A subset $B$ of $\mathbf{A}$ is said to be absorbing if $\omega^{\mathbf{A}}\left(a_{0}, \ldots, a_{m-1}\right) \in B$ whenever $a_{i} \in B$ for some $i<m$. 
THEOREM 7.10. Assume $\mathbf{A}$ is a finite, nontrivial algebra.

(1) $Q v(\mathbf{E t} \mathbf{A})$ is finitely based.

(2) Assume further that $\{0,1\}$ is an absorbing subset of $\mathbf{A}$ that does not include the range of any term function of $\mathbf{A}$. Then, if $V a \mathbf{A}$ is not finitely based, neither is $V a(\mathbf{E t} \mathbf{A})$.

PROOF. (1) By Theorems I and 7.9(1).

(2) Let $\kappa\left(x_{0}, \ldots, x_{m-1}\right) \approx \lambda\left(x_{0}, \ldots, x_{m-1}\right)$ be any identity of Et $\mathbf{A}$. The additional hypothesis of (2) assures that, if $\kappa$ contains any occurrence of one of the adjoined operations symbols $+, \cdot,-, 0,1$, or $e q$, then $\lambda$ must also contain such an occurrence. For suppose otherwise. Choose $a_{0}, \ldots, a_{m-1} \in A$ such that $\lambda^{\mathbf{A}}\left(a_{0}, \ldots, a_{m-1}\right) \notin\{0,1\}$. Since $\{0,1\}$ is absorbing, $\kappa^{\mathbf{A}}\left(a_{0}, \ldots, a_{m-1}\right) \in\{0,1\}$, contrary to the assumption that $\kappa \approx \lambda$ is an identity of $\mathbf{A}$.

Since $\mathbf{A}$ is a reduct of $\mathbf{E t} \mathbf{A}$, the identities of $\mathbf{A}$ coincide with the identities of Et $\mathbf{A}$ that contain none of the symbols $+, \cdot,-, 0,1$, or eq. Let $\kappa \approx \lambda$ be such an identity. Then by the remark of the previous paragraph, only axioms with the same property can be used in any derivation of $\kappa \approx \lambda$ of minimal length in one of the standard deductive systems of equational logic. Thus any base for $V a(\mathbf{E t} \mathbf{A})$ must include a base for $V a \mathbf{A}$.

It is easy to construct finite algebras satisfying the hypotheses of $7.10(2)$. Let $\mathbf{B}$ be any finite algebra such that $V a \mathbf{B}$ is not finitely based, and, in addition, $\mathbf{B}$ is regular in the sense that, for every identity $\kappa \approx \lambda$ of $\mathbf{B}$, each variable occurring in $\kappa$ also occurs in $\lambda$, and vice-versa. (Most of the known algebras that generate nonfinitely based varieties have this property; see for instance McNulty and Shallon [24].) Let $\mathbf{A}$ be the algebra over the same language obtained from $\mathbf{B}$ by adjoining new elements 0 and 1 , and extending the fundamental operations by setting $\omega^{\mathbf{A}}\left(a_{0}, \ldots, a_{m-1}\right)=0$ if $0 \in\left\{a_{0}, \ldots, a_{m-1}\right\}$, and $\omega^{\mathbf{A}}\left(a_{0}, \ldots, a_{m-1}\right)=1$ if $0 \notin\left\{a_{0}, \ldots, a_{m-1}\right\}$ but $1 \in\left\{a_{0}, \ldots, a_{m-1}\right\}$. It is easy to see that, because $\mathbf{B}$ is regular, $V a \mathbf{A}=V a \mathbf{B}$. Thus $\mathbf{A}$ satisfies the hypotheses of 7.10(2). This gives a large number of finite algebras whose quasi-identities are finitely based but whose identities are not.

Because $\mathcal{G E} T$ is finitely based, we could have used one of the corollaries of Theorem 3.1 to show $Q v(\mathbf{E t} \mathbf{A})$ is finitely based instead of the much deeper Theorem I. The result can also be obtained from a theorem of Czelakowski and Dziobiak [12] (Theorem 9.2 below) since $Q v(\mathbf{E t} \mathbf{A})$ turns out to have REDPM. From the fact that

$$
\mathcal{G E} \tau_{\mathrm{RFSI}} \vDash \forall x y z w(e q(x, y)+e q(z, w) \approx 1 \rightarrow x \approx y \vee z \approx w),
$$

we can conclude that principal $\mathcal{G} \mathcal{E} T$-congruence meets are definable by the nonparameterized equation $e q(x, y)+e q(z, w) \approx 1$, and hence $\mathcal{G} \mathcal{E} T$ and all of its relativized subvarieties have REDPM.

The original proof of $7.10(1)$ is still of value however since it can be generalized to give natural examples of finitely generated quasivarieties that have all the essential properties of the quasivariety $Q v(\mathbf{E t} \mathbf{A})$ mentioned in Theorem 7.10 but fail to have REDPM. $Q v(\mathbf{E t} \mathbf{A})$ is relatively congruence-distributive and has REDPM essentially because the variety of Boolean algebras has these properties. The idea is to replace the Boolean value algebra in the construction of $Q v(\mathbf{E t} \mathbf{A})$ by another value algebra that generates a variety with the first of these properties but not 
the second. The actual construction will appear elsewhere. A wide class of nonBoolean equality-test algebras with various interesting properties can be obtained in this way.

8. Data structures and abstract data types. Heterogeneous (many-sorted) Boolean test algebras, as previously remarked, arise naturally in the theory of data structures. Moreover, the theory of quasivarieties of homogeneous algebras developed in the previous sections can be extended mutatis mutandis to this more general context. Thus the quasi-identities of every finite heterogeneous equality-test algebra are finitely based, and, more generally, we have heterogeneous analogues of both Theorems I and II. ${ }^{3}$

Let $\mathbf{S}$ be any algebra (homogeneous or heterogeneous). A term in the language of $\mathbf{S}$ without variables is called a ground term. By a ground-term function of $\mathbf{S}$ we mean any element of $S$ of the form $\kappa^{\mathbf{S}}$ where $\kappa$ is a ground term of $\mathbf{S}$. A data structure is any heterogeneous algebra $\mathbf{S}$ with the property that every element is the value of a ground-term function. By an abstract data type we mean the isomorphism class of of some data structure. Our treatment of abstract data types follows Meseguer and Goguen [25] with some minor exceptions; see also Goguen, Thatcher, and Wagner [17].

To illustrate the application of our results to data structures we consider algebras of stacks. A stack algebra consists of three sorts: the first, the stack elements, can be any homogeneous algebra over an arbitrary language type (signature) $T$ that is a data structure (i.e., every element is the range of a ground-term function). The stacks themselves form the second sort. They consist of all finite sequences of stack elements, possibly with some fixed upper bound on their length. There are two intra-sort operations on stacks: the nullary empty stack and the unary pop. The empty stack denotes the empty sequence, and pop applied to the stack $\left\langle a_{0}, \ldots, a_{n-1}\right\rangle$ returns the new stack $\left\langle a_{0}, \ldots, a_{n-2}\right\rangle$ (unless the original stack is empty). The third sort is the two-element Boolean algebra with the usual Boolean operations.

In addition to the intra-sort operations there are five inter-sort operations, full, $p u s h, t o p, e e q$, and seq. full applied to a stack of maximal allowable length returns the Boolean value 1; otherwise it returns 0. push applied to a stack $\left\langle a_{0}, \ldots, a_{n-1}\right\rangle$ and an element $b$ returns the stack $\left\langle a_{0}, \ldots, a_{n-1}, b\right\rangle$ (unless the original stack is full). top applied to $\left\langle a_{0}, \ldots, a_{n-1}\right\rangle$ returns the element $a_{n-1}$ (unless the stack is empty). eeq is the element equality test; applied to a pair of elements it returns 1 or 0 depending on whether the elements are equal or not. The stack equality test $s e q$ is similarly defined.

We have not specified the value of the operations for all possible valid input values. For instance, $p o p(s)$ has not been defined when $s$ in the empty stack, and similarly for $\operatorname{push}(s, e)$ when $s$ is full. The operations can be left undefined on these inputs, and stack algebras treated as partial algebras. Another possibility is to introduce a new error constant for each sort. The set of error values will act as an absorbing set with regard to the operations of the algebra, and, where previously undefined, the operations will now return the error value of the appropriate sort.

\footnotetext{
${ }^{3}$ Heterogeneous or many-sorted algebras were first considered by Birkhoff and Lipson [6] and Higgins [18]. Their equational metatheory is developed in Goguen and Meseguer [16].
} 
Terms in the language of stack algebras are divided into element, stack, and Boolean terms. Equations are defined only when both sides are of the same sort; quasi-equations are defined the usual way. It is easy to see that in any stack algebra each stack is the value of a term that involves only push, empty stack, and ground element terms. Thus every stack algebra is a data structure.

The quasivariety of generalized stack algebras is generated by all stack algebras of a given type. It is finitely based and relatively congruence-distributive; cf. [30]. The heterogeneous version of Theorem I can be applied to give the following analogue of 7.10 .

THEOREM 8.1. (1) The quasi-identities of every finite stack algebra are finitely based.

(2) If the identities of the element-sort algebra of a stack algebra are not finitely based, then neither are the identities of the stack algebra.

In part (2) we need no special assumptions about the element-sort algebra like those required in $7.10(2)$. Since there is no operation that maps either the stack or Boolean sort into the element sort, every element-sort identity is pure in the sense that its terms involve only the internal operations of the sort. Thus any derivation of a (pure) element-sort identity can involve only identities of the same kind, and hence any base for the identities of a stack algebra must include a base for the identities of its element-sort algebra. This gives a slightly stronger result than $8.1(2)$.

(3) If the identities of the element-sort algebra of a stack algebra are not finitely based, then they are not derivable from any finite set of identities of the stack algebra.

Heterogeneous equality-test algebras are more natural than their homogenous counterpart. As mentioned previously homogeneous equality-test algebras are most naturally thought of as heterogeneous algebras in which the various sorts are collapsed into one. This introduces the possiblity of unintended interaction between operations of different sorts that must be accounted for by contrived hypotheses like those found in 7.9(2) and 7.10(2).

Finite homogeneous algebras whose identities are not finitely based, and in which each element is the value of a ground-term function, are known to exist (see for instance [29]). Taking one of these for the element-sort algebra we get a finite stack algebra $\mathbf{S}$ whose quasi-identities are finitely based while its pure element-sort identities fail to be logical consequences of any finite set of identities of $\mathbf{S}$.

Specification of abstract data types. We end this section with some remarks on the specification problem for abstract data types. Let $\mathbf{S}$ be a data structure. By a ground identity of $\mathbf{S}$ we mean any identity of $\mathbf{S}$ that involves only ground terms, i.e., that does not contain any variables. The abstract data type of $\mathbf{S}$ is completely determined by the set of ground identities of $\mathbf{S}$. Following [17] we say that a set $\Gamma$ of equations or quasi-equations is a specification, or more precisely a correct specification, of the data structure $\mathbf{S}$ and its abstract data type if $\mathbf{S}$ is the initial algebra of the variety or quasivariety defined by $\Gamma$. This is equivalent to saying that each member of $\Gamma$ is an identity or quasi-identity of $\mathbf{S}$, and that every ground identity of $\mathbf{S}$ is a logical consequence of $\Gamma$; see [25, Theorem 18]. $\Gamma$ is 
called a equational specification if it consists exclusively of identities; otherwise it is a conditional specification.

A data structure will have many specifications that are not logically interderivable. For instance, any base for the identities of $\mathbf{S}$ is a specification, as is any base for its quasi-identities, but in general the quasi-identities of $\mathbf{S}$ are not derivable from its identities. A finite base for the quasi-identities of $\mathbf{S}$ will give a finite conditional specification, but $\mathbf{S}$ may have a finite conditional specification without having a finite base for its quasi-identities.

Correct specifications are important in the theory of data structures because, in completely characterizing the abstract data type of a structure, they provide a means of precisely specifying its essential properties independent of a particular implementation. This is widely viewed as one of the first steps in the modularization of a large-scale software development. At the implementation level correct specifications are used to verify the correctness of a particular implementation. They themselves may also serve as the means of implementation via the deductive apparatus of equational or quasi-equational logic; see for instance O'Donnell [27]. The merit of a specification is judged on the basis of how well it serves these purposes. A rough criterion is size, but there may be good reasons for choosing a particular specification over a smaller one. For instance a more complicated conditional specification, that is complete in the sense that it forms a base for all quasi-identities of the data structure, may be desirable because it leads to a more efficient algorithm for deducing ground identities.

It seems likely that the sets of ground identities, identities, and quasi-identities can be finitely based or nonfinitely based in every conceivable combination. For instance the ground identities of a finite data structure are always finitely based, while, as the last theorem and the remarks following it show, there exist finite data structures whose quasi-identities are finitely based while their identities are not.

A related problem concerns the relative strength of conditional and equational specification. Thatcher, Wagner, and Wright [34] show that the former is more powerful by giving a simple example of a computable abstract data type with a finite conditional specification but no finite equational one. We give another example of such a data structure as an application of Theorem 8.1. It is more complicated than the one in [34], but it may be of some intrinsic interest since it is a generalized stack algebra.

Let $\mathbf{S}$ be a finite stack algebra whose quasi-identities are finitely based, while at the same time its pure element-sort identities are not a consequence of any finite set of identities of $\mathbf{S}$. Let $\Gamma$ be a finite base for the quasi-identities of $\mathbf{S}$. Since $\mathbf{S}$ is finite it has a finite equational specification, and we must replace it by another data structure. Let $\mathbf{F}$ be an algebra in the variety $\mathrm{Va} \mathbf{S}$ that is freely generated by an infinite number of elements from its element-sort domain. Extend the language (signature) of $\mathbf{S}$ by adjoining a new nullary operation symbol of the element sort for each free generator of $\mathbf{F}$, and let $\mathbf{F}^{+}$be the algebra over the extended language obtained from $\mathbf{F}$ by having each new nullary symbol denote its associated free generator. $\mathbf{F}^{+}$is clearly a data structure. (It is not a stack algebra since its Boolean-sort algebra is in general an infinite Boolean algebra; it is of course a generalized stack algebra.) 
THEOREM 8.2. The abstract data type of $\mathbf{F}^{+}$fails to have a finite equational specification, but it does have a finite conditional specification.

The proof is straightforward and will be omitted. It is based on the fact that any ground identity of $\mathbf{F}^{+}$can be converted into an identity of $\mathbf{S}$ by substituting variables for the new nullary symbols, and vice-versa.

One unsatisfactory feature of the data structure $\mathbf{F}^{+}$is the infinite set of nullary element-sort operations in its language. This can be avoided by adding a fourth sort of natural numbers and injecting them into the element-sort to play the role of the free generators.

For a more detailed discussion of the axiomatization and specification of heterogeneous equality-test and related data structures see [30].

9. Connections with previous work and open problems. The first example of a finite algebra that fails generate a finitely based quasivariety was given by $\mathrm{Ol}^{\prime}$ sanskiı̌ [28]. Subsequently many other examples of this kind were found, even among members of congruence-distributive varieties: for example lattices (Belkin [4], Tumanov [35]), Heyting algebras (Dziobiak [14]), and interior algebras (Rybakov [31]). Moreover, complete characterizations have been given of finite groups ( $\mathrm{Ol}^{\prime}$ `anskiı̌ [28]) and finite rings (Belkin [4]) that generate nonfinitely based quasivarieties, and a partial characterization of finite semigroups with this property has also been obtained (Sapir [32]).

The first result in this area of a general algebraic character was obtained by Blok and Pigozzi [8] by generalizing the method of Rybakov. It applies only to subquasivarieties of varieties with EDPM (see $\S 7$ ). Examples of varieties with EDPM are distributive lattices, Boolean algebras, relation algebras, cylindric algebras of finite dimension, and any discriminator variety. Among varieties with EDPM that are not discriminator varieties are Heyting algebras, interior algebras, vector groups, $F$-rings, and vector lattices. Varieties with EDPM were first studied by Baker [1]. He proved that if $\mathcal{V}$ is a congruence-distributive variety with this property, and $K$ is an elementary subclass defined by a universal closure of a disjunction of equations (a UDE in Baker's terminology), then $\mathcal{K}$ generates a finitely based variety relative to $\mathcal{V}$. An easy corollary of this result is that every finitely generated congruencedistributive variety with EDPM is finitely based. Subsequently it was shown in [8] that every variety with EDPM is congruence-distributive, and thus that every finitely generated variety with EDPM is finitely based. The following theorem is the main result of $[8]$.

THEOREM 9.1. Let $\mathcal{Q}$ be a subquasivariety of a variety $\mathcal{V}$ with EDPM. If $\mathcal{Q}$ is generated by a finite set of finite, subdirectly irreducible members of $\mathcal{V}$, then $\mathcal{Q}$ is finitely based.

Czelakowski and Dziobiak [12] improved this result in several ways, and greatly clarified its relationship to Baker's theorem. Moreover their work led directly to the discovery of Theorem I. The key to the relationship is the relativization of the EDPM property to quasivarieties. They obtained the following natural generalization of the finite basis result in Baker [1]. Recall that a class of algebras is strictly elementary if it is definable by a finite set of first-order sentences. 
THEOREM 9.2 [12]. Let $\mathcal{Q}$ be a quasivariety with REDPM. If $\mathcal{Q}_{\mathrm{RFSI}}$ is strictly elementary, in particular, if $\mathcal{Q}$ is finitely generated, then $\mathcal{Q}$ is finitely based.

If $\mathcal{Q}$ is a finitely generated subquasivariety of a variety with EDPM, Czelakowski and Dziobiak proved that $\mathcal{Q}$ satisfies the hypothesis of Theorem 9.1 just in case it has REDPM; see Theorem 7.4. Thus they were able to obtain 9.1 as an immediate corollary of 9.2 and 7.4. These latter two theorems led naturally to the conjecture of Theorem I. Dziobiak was able to prove the following weaker result.

THEOREM 9.3 [15]. Every finitely generated and relatively congruence-distributive quasivariety that is included in a congruence-distributive variety is finitely based.

Theorem 8.1 can also be obtained as an immediate consequence of 9.3 and the fact every variety with EDPM is congruence-distributive.

Connections with logic. The various relativized notions that we have considered in this paper, such as relative congruence-distributivity, relative equationally definable principal congruence meets, and relative subvariety, have their origin in logic. And many of the theorems involving these notions first appeared in metalogical form. For example Theorems II and 9.2 are the algebraic algebraic analogues of metalogical results appearing in [9 and 10], respectively. ${ }^{4}$

A deductive system is a pair $S=\langle T, \vdash\rangle$ consisting of a language type $T$ and a consequence relation $\vdash$ between the formulas of type $T$ that satisfies certain well known conditions (see Tarski [33]). A deductive system is usually defined by logical axioms and inference rules, for example, modus ponens: $x, x \rightarrow y \vdash y$, and the rule of necessitation of modal logic: $x \vdash \square x$. Any set of formulas that includes the logical axioms and is closed under substitution and the inference rules is called a logic over S. A logic is often defined by a set of nonlogical axioms.

Deductive systems and logics can also be defined semantically. A matrix consists of an algebra $\mathbf{A}$ of the same language type as $S$, together with a set of designated elements $D$. A matrix $\langle\mathbf{A}, D\rangle$ is a model of $S$ if $D$ is closed under the consequence relation $\vdash$ in the natural sense. Any class of matrices of type $T$ defines a deductive system $S=\langle T, \vdash\rangle$, where $\vdash$ is the largest consequence relation such that each of the matrices is a model of $S$. Similarly, every class of matrix models of $S$ defines a logic over $S$. For any algebra $\mathbf{A}$ of the same language type as a deductive system $S$, a set $D$ of designated elements is a $S$-filter if $\langle\mathbf{A}, D\rangle$ is a matrix-model of $S$. The set of all $S$-filters on $\mathbf{A}$ forms an algebraic lattice. $S$ is filter-distributive if this lattice is distributive for every algebra $\mathbf{A}$.

There is an obvious analogy between quasivarieties and deductive systems (more precisely, their matrix-model classes), and between relative subvarieties and logics. Quasi-identities correspond to consequence relationships of the form $\Gamma \vdash \kappa$, and identities to theorems (i.e., formulas $\kappa$ such that $\vdash \kappa$ ). The finite axiomatizability problem for a finite matrix takes two forms. One can ask if the deductive system it defines is finitely axiomatizable. This corresponds to the finite basis problem for the quasivariety generated by a finite algebra. If the matrix is a model of a

\footnotetext{
${ }^{4}$ Propositional logic is the part of logic most closely connected to theory of varieties and quasivarieties. For a comprehensive account of much of the modern work on propositional logic see Wójcicki $[\mathbf{3 7}, \mathbf{3 8}]$.
} 
deductive system $S$, one can ask if the logic it defines is finitely axiomatizable, i.e., if its theorems are logical consequences of a finite set of logical and extra-logical axioms and inference rules. This corresponds to the finite basis problem for the relative subvariety generated by a finite member of a quasivariety $\mathcal{Q}$. Both kinds of problems have been considered in the literature, but the finite axiomatizability problem for logics is the more important from a historical point of view.

A logic defined by a finite matrix is called tabular. The finite axiomatizability of tabular logics was first considered in connection with many-valued logics, and the first result of a general character in this area was due to Wajsberg [36]; see Lukasiewicz and Tarski [21]. Let $S$ be any deductive system with $\rightarrow$ and $\neg$ as the only connectives and modus ponens as the only rule of inference. Wajsberg proved that a sufficient condition for a tabular logic over $S$ to be finitely axiomatizable is that it include among its theorems four relatively simple formulas; these turn out to be theorems of a large class of logics that contains in particular all the many-valued $\operatorname{logics} L_{n}$ for $n$ finite that were considered in Lukasiewicz and Tarski [21]. Two recent results of a similar character lead directly to Theorem II. Blok and Köhler [7] use a matrix-theoretic version Jónsson's lemma [19] to prove that every quasinormal tabular modal logic is finitely axiomatizable. This result is generalized and extended in Blok and Pigozzi [9] into a metalogical analogue of Baker's Theorem [2] that applies to a wide class of deductive systems called protoalgebraic systems. Recall that a deductive system $S$ is said to be filter-distributive if the lattice of $S$-filters of every algebra is distributive.

THEOREM 9.4 [9]. Every tabular logic in a filter-distributive protoalgebraic deductive system is finitely axiomatizable provided $S$ has only finitely many inference rules.

Theorem II is the natural algebraic analogue of this result under the correspondence between $\mathbf{Q}$-congruences and $\boldsymbol{S}$-filters. Corollary 3.3 is actually a closer analogue of 9.4. Theorem II is a stronger result than 3.3 because of the absence of the assumption that $\mathcal{Q}$ is finitely based. However the corresponding strengthening of Theorem 9.4. can also be shown to hold: every tabular logic in a filter-distributive protoalgebraic deductive system $S$ is finitely axiomatizable, regardless of whether $S$ itself is finitely axiomatizable.

A deductive system is said to be disjunctive if there exists a finite system $\delta_{0}(p, q), \ldots, \delta_{m-1}(p, q)$ of formulas in two variables that collectively act like a disjunction connective: for any set $\Gamma$ of formulas,

$$
\Gamma, \varphi \vdash \vartheta \text { and } \Gamma, \psi \vdash \vartheta \quad \text { iff } \quad \Gamma \cup\left\{\delta_{i}(\varphi, \psi): i \leq m\right\} \vdash \vartheta
$$

for all formulas $\varphi, \psi$, and $\vartheta$. Dzik and Suszko [13] proved that every disjunctive deductive system is filter-distributive. Disjunctive deductive systems correspond to quasivarieties with REDPM in a natural way, and the above result suggests that that every variety with EDPM is congruence-distributive. This result was proved in $[9]$.

Similarly, Theorem 9.2 is the algebraic analogue of the following meta-logical result of Czelakowski [11]. a matrix model $\langle\mathbf{A}, D\rangle$ of a deductive system $S$ is prime if $D$ is finitely meet-irreducible in the lattice of $S$-filters of $\mathbf{A}$. 
THEOREM 9.5 [11, Theorem III.2]. Let $S$ be a disjunctive deductive system. If the class of prime matrix models of $S$ is strictly elementary, then $S$ is finitely axiomatizable.

\section{Open problems.}

PROBLEM 9.6. Assume $\mathcal{Q}$ is a relatively congruence-distributive quasivariety such that $\mathcal{Q}_{\mathrm{RFSI}}$ is strictly elementary. Is $\mathcal{Q}$ necessarily finitely based?

By Theorem I the answer is positive if $\mathcal{Q}$ is finitely generated, and by Theorem 9.2 the answer is positive when $\mathcal{Q}$ has REDPM. We can raise a similar problem for relative subvarieties.

PROBLEM 9.7. Assume $R$ is a relative subvariety of a relatively congruencedistributive quasivariety $\mathcal{Q}$, and that $R_{\mathrm{RFSI}}$ is strictly elementary. Is $\mathcal{R}$ necessarily finitely based?

A positive solution to this problem would not be an easy consequence of a positive solution to 9.6 in the same way that Theorem II is an easy corollary of Theorem I.

In [9] the question was raised if Theorem 9.1 continues to hold when the premiss that $\mathcal{V}$ has EDPM is weakened to the requirement that $\mathcal{V}$ is congruence-distributive or has equationally definable principal congruences. (See [9] for the definition of this latter notion.) Czelakowski and Dziobiak [12] have shown that this is not true in the congruence-distributive case by constructing a finite, subdirectly irreducible lattice whose generated quasivariety is not finitely based. The other problem is still open.

PROBLEM 9.8. Let $\mathcal{Q}$ be a subquasivariety of a variety $\mathcal{V}$ with equationally definable principal congruences. If $\mathcal{Q}$ is generated by a finite set of finite, subdirectly irreducible members of $\mathcal{V}$, is $\mathcal{Q}$ necessarily finitely based?

The following interesting problem was personally communicated to the author by A. Wroński.

PROBLEM 9.9. Is it true that, for any finite algebra $\mathbf{A}$, there exists a finitely based quasivariety $\mathcal{Q}$ such that $V a \mathbf{A}=V a \mathcal{Q}$ ?

In syntactical terms the problem asks if it is true that, for every finite algebra $\mathbf{A}$, there exists a finite set of quasi-identities $\Gamma$ such that the identities of $\mathbf{A}$ coincide with the identities derivable from $\Gamma$. Wronski has verified that this is true for a number of the examples in the literature of finite algebras whose identities are not finitely based. On the other hand the corresponding problem for finite matrices is known to have a negative solution; see Wojtylak [39].

The following closely related problem comes up naturally in connection with Theorem 7.10.

ProBlem 9.10. Does there exist a finite algebra whose identities and quasiidentities both fail to be finitely based, but whose identities are all consequences of a finite set of its quasi-identities?

In problem 9.9 we can replace the finite algebra $\mathbf{A}$ by a finite set $\mathcal{M}$ of finite algebras and get a formulation of the problem that is easily seen to be equivalent to the original. Among the quasivarieties $\mathcal{Q}$ with the property that $V a \mathcal{M}=V a \mathcal{Q}$ 
there is a largest one $(V a \mathcal{M})$ and a smallest one $(Q v \mathbf{F}$, where $\mathbf{F}$ is the free algebra over $V a \mathcal{M}$ with an infinite number of free generators). Problem 9.9 asks if there is at least one finitely based quasivariety in this interval. We can also ask if there exists such a quasivariety in the smaller interval [Qv $\mathcal{M}, V a \mathcal{M}]$.

PROBLEM 9.11. Is it true that, for every finite set $\mathcal{M}$ of finite algebras, there exists a finitely based quasivariety $\mathcal{Q}$ such that $Q v \mathcal{M} \subseteq \mathcal{Q}$ and $V a \mathcal{M}=V a \mathcal{Q}$ ?

It seems unlikely that this problem will have a positive solution. First of all, such a solution would imply that there exist no finitely generated quasivarieties that are inherently nonfinitely based. A locally finite quasivariety (variety) is inherently nonfinitely based if it is not included in any finitely based and locally finite quasivariety (variety). There are many examples of finitely generated varieties that are inherently nonfinitely based. See McNulty and Shallon [24] and Baker, McNulty, and Werner [3].

Secondly, it would provide a positive answer, in a very strong sense, to a problem of B. Jónsson quoted in McKenzie [22]:

PROBLEM 9.12. Is it true that, for every finite set $\mathcal{M}$ of finite algebras, $Q v \mathcal{M}=$ Va $\mathcal{M}$ implies $V a \mathcal{M}$ is finitely based?

Baker's theorem would be an immediate consequence of Jónsson's lemma and a positive solution of this problem. Finally we mention the problem of extending Theorem I to relatively congruence-modular quasivarieties. It would give a natural generalization of a recent result of McKenzie [23]. He has shown that every finitely generated, residually finite congruence-modular variety is finitely based. This can be rephrased in the following way: every congruence-modular variety that is finitely generated as a quasivariety is finitely based.

PROBLEM 9.13. Is it true that every finitely generated and relatively congruencemodular quasivariety is finitely based?

\section{REFERENCES}

1. K. Baker, Primitive satisfaction and equational problems for lattices and other algebras, Trans. Amer. Math. Soc. 190 (1974), 125 150.

2. __ Finite equational basis for finite algebras in a congruence-distributive equational class, Adv. in Math. 24 (1977), 207-243.

3. K. Baker, G. McNulty and H. Werner, The finitely based varieties of graph algebras, Acta Sci. Math. (Szeged) 51 (1987), 3-15.

4. V. P. Belkin, Quasi-identities of finite rings and lattices, Algebra i Logika 17 (1978), 247-259. (Russian)

5. G. Birkhoff, Subdirect unions in universal algebra, Bull. Amer. Math. Soc. 50 (1944), 764-768.

6. G. Birkhoff and J. D. Lipson, Heterogeneous algebras, J. Combinatorial Theory 8 (1970), 115-133.

7. W. J. Blok and P. Köhler, Algebraic semantics for quasi-classical modal logics, J. Symbolic Logic 48 (1983), 941-964.

8. W. J. Blok and D. Pigozzi, A finite basis theorem for quasivarieties, Algebra Universalis 22 (1986), 1-13.

9. , Protoalgebraic logics, Studia Logica 45 (1986), 331-363.

10. S. Burris and H. P. Sankappanavar, A course in universal algebra, Springer-Verlag, New York, 1981.

11. J. Czelakowski, Matrices, primitive satisfaction, and finitely based logics, Studia Logica 42 (1983), 89-104. 
12. J. Czelakowski and W. Dziobiak, Congruence distributive quasivarieties whose finitely subdirectly irreducible members form a universal class, Preprint.

13. W. Dzik and R. Suszko, On distributing of closure systems, Polish Acad. Sci. Inst. Philos. Sociol. Bull. Sect. Logic 6 (1977), 64-66.

14. W. Dziobiak, Concerning axiomatizability of the quasivariety generated by a finite Heyting or topological Boolean algebra, Studia Logica 41 (1982), 415-428.

15. __ Finitely generated congruence distributive quasivarieties of algebras, Preprint.

16. J. A. Goguen and J. Meseguer, Completeness of many-sorted equational logic, Houston J. Math. 11 (1985), 307-334.

17. J. A. Goguen, J. W. Thatcher, and E. Wagner, An initial algebra approach to the specification, correctness, and implementation of abstract data types, Current Trends in Programming Methodology, R. Yeh, ed., Prentice-Hall, 1978, pp. 80-149.

18. P. J. Higgins, Algebras with a scheme of operators, Math. Nachr. 27 (1963), 115-132.

19. B. Jonsson, Algebras whose congruence lattices are distributive, Math. Scand. 21 (1967), 110121.

20. P. Köhler, Brouwerian semilattices, Trans. Amer. Math. Soc. 268 (1981), 103-126.

21. J. Lukasiewicz and A. Tarski, Investigations into the sentential calculus, A. Tarski, Logic, Semantics, and Metamathematics, 2nd ed., Hackett, Indianapolis, Indiana, 1983.

22. R. McKenzie, $A$ finite algebra $A$ with $S P(A)$ not elementary, Algebra Universalis 8 (1978), 5-7.

23. __ Finite equational bases for congruence modular algebras, Preprint.

24. G. F. McNulty and C. Shallon, Inherently nonfinitely based finite algebras, Universal Algebra and Lattice Theory, R. Freese and O. Garcia, eds., Lecture Notes in Math., vol. 1004, Springer-Verlag, Berlin, 1983, pp. 206-231.

25. J. Meseguer and J. A. Goguen, Initiality, induction, and computability, Algebraic Methods in Semantics, M. Nivat and J. Reynolds, eds., Cambridge Univ. Press, 1985, pp. 459-540.

26. W. Nemitz, Implicative semi-lattices, Trans. Amer. Math. Soc. 117 (1965), 128-142.

27. M. J. O'Donnell, Equational logic as a programming language, The MIT Press, Cambridge, Mass., 1985.

28. A. J. Ol'sanskiř, Varieties of finitely approximable groups, Izv. Akad. Nauk SSSR Ser. Mat. 33 (1969), 915-927.

29. D. Pigozzi, Minimal, locally finite varieties that are not finitely axiomatizable, Algebra Universalis 9 (1979), 374-390.

30. __ Equality-test and if-then-else algebras: Axiomatization and specification, Preprint.

31. V. V. Rybakov, Bases of quasivarieties of finite modal algebras, Algebra i Logica 21 (1982), 219-227. (Russian)

32. M. V. Sapir, On the quasivarieties generated by finite semigroups, Semigroup Forum 20 (1980), 73-88.

33. A. Tarski, Fundamental concepts of the methodology of the deductive sciences, Logic, Semantics, and Metamathematics, 2nd ed., Hackett, Indianapolis, Indiana, 1983.

34. J. W. Thatcher, E. G. Wagner and J. B. Wright, Data type specification: parameterization and the power of specification techniques, ACM Trans. Program. Lang. Syst. 4 (1982), 711-732.

35. V. I. Tumanov, On finite lattices not having independent basis of quasi-identities, Mat. Zametki 36 (1984), 625-634. (Russian)

36. M. Wajsberg, Beiträge sum Metaaussagenkalkül I, Monatsh. Math. Phys. 42 (1935), 221-242.

37. R. Wojcicki, Lectures on propositional calculi, Publ. House Polish Acad. Sci., The Polish Academy of Sciences Institute of Philosophy and Sociology, Ossolineum, Warsaw, 1984.

38. _ Theory of logical calculi. An introduction, Manuscript.

39. P. Wojtylak, Strongly finite logics: finite axiomatizability and the problem of the supremum, Polish Acad. Sci. Inst. Philos. Sociol. Bull. Sect. Logic 8 (1979), 99-111.

\section{Department of Mathematics, Iowa State University, Ames, Iowa 50011}

